\title{
AIP Low Temperature Physics
}

Structure and vibrational spectra of gauche- and trans-conformers of ethanol: Nonempirical anharmonic calculations and FTIR spectra in argon matrices

G. A. Pitsevich, I. Yu. Doroshenko, V. Ye. Pogorelov, V. Sablinskas, and V. Balevicius

Citation: Low Temp. Phys. 39, 389 (2013); doi: 10.1063/1.4801995

View online: http://dx.doi.org/10.1063/1.4801995

View Table of Contents: http://tp.aip.org/resource/1/LTPHEG/v39/i4

Published by the AIP Publishing LLC.

Additional information on Low Temp. Phys.

Journal Homepage: http://tp.aip.org/

Journal Information: http://ltp.aip.org/about/about_the_journal

Top downloads: http://ltp.aip.org/features/most_downloaded

Information for Authors: http://tp.aip.org/authors

\section{ADVERTISEMENT}

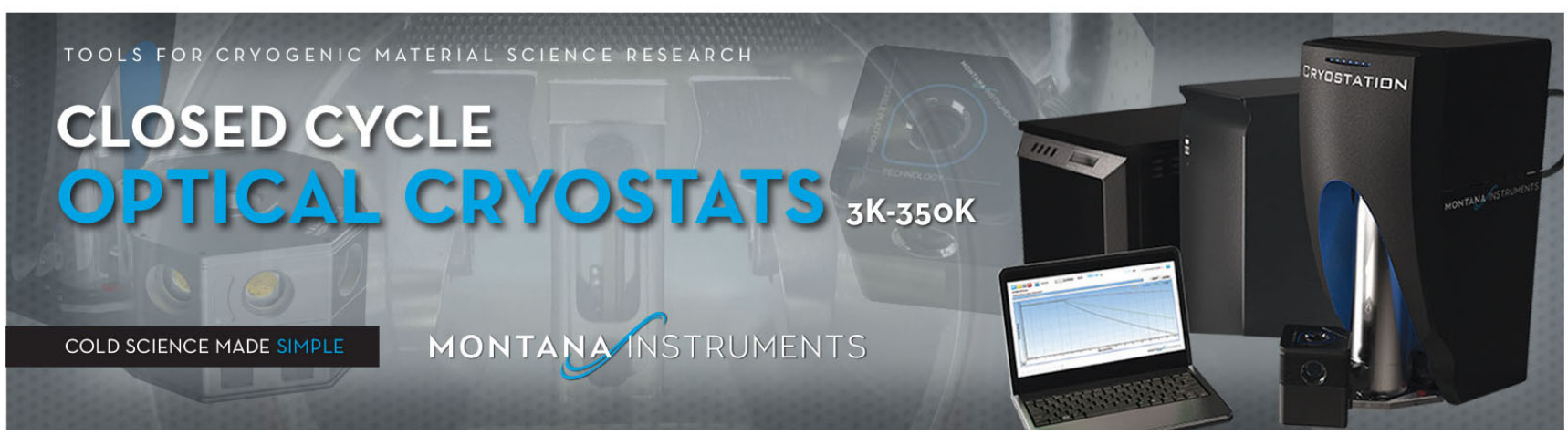




\title{
LATTICE DYNAMICS
}

\section{Structure and vibrational spectra of gauche- and trans-conformers of ethanol: Nonempirical anharmonic calculations and FTIR spectra in argon matrices}

\author{
G. A. Pitsevich \\ Physics Faculty, Belarusian State University, pr. F. Skoriny 4, Minsk 220050, Belarus \\ I. Yu. Doroshenko a) and V. Ye. Pogorelov \\ Physics Faculty, Taras Shevchenko Kiev National University, pr. Glushkova 4, Kiev 03022, Ukraine \\ V. Sablinskas and V. Balevicius
}

Physics Faculty, Vilnius University, Sauletekio St. 9-3, Vilnius 10222, Lithuania (Submitted June 8, 2012; revised July 23, 2012)

Fiz. Nizk. Temp. 39, 499-513 (April 2013)

\begin{abstract}
Low-temperature infrared absorption spectra are obtained for ethanol isolated in an argon matrix at temperatures of $20-45 \mathrm{~K}$ range for ratios of the numbers of the molecules being studied to the numbers of matrix atoms of 1:1000 and 1:2000. A preliminary interpretation of the spectra is obtained on the basis of the temperature variations in the spectra and published data. The structure of the ethanol conformers, rotational constants, and internal rotation barriers of the methyl and hydroxyl groups are calculated in the B3LYP/cc-pVQZ approximation. The harmonic and anharmonic IR spectra of the gauche- and trans-conformers are calculated in the same approximation. The force fields of the two conformers and the distributions of the potential energy of the normal vibrations are calculated and compared for a general set of dependent coordinates. Anharmonicity effects are taken into account by introducing spectroscopic masses for the hydrogen atoms when calculating the normal vibrations in the harmonic approximation. (C) 2013 AIP Publishing LLC [http://dx.doi.org/10.1063/1.4801995]
\end{abstract}

\section{Introduction}

Alcohol molecules are of interest to researchers because of their ability to form various cluster structures through the formation of intermolecular hydrogen bonds. Studies of these structures can, in principle, make it possible to follow the transition from single molecules in a gaseous medium to liquid media, as well as the change in the spectral characteristics along this path. ${ }^{1,2}$ The largest number of these studies concerns the methanol molecule, as it is the simplest representative of the alcohols. ${ }^{3}$ In this molecule, as in all the alcohols, the hydroxyl group can undergo an internal rotation relative to the $\mathrm{C}-\mathrm{O}$ bond. ${ }^{4-6}$ The ethanol molecule has additional possibilities beyond those of methanol. The $\mathrm{CH}_{3} \mathrm{CH}_{2} \mathrm{OH}$ molecule has two tops: symmetric methyl and asymmetric hydroxyl. In addition, ethanol can exist in two conformations: trans-, where the molecule has a $C_{S}$ symmetry, and gauche, where there are no elements of symmetry. These properties have led to great interest in studying its properties; this interest became greater once it was established ${ }^{7-10}$ that the ethanol molecule exists in dense, hot interstellar molecular gas clouds.

Progress in understanding the structure and a number of physical properties of the ethanol molecule has depended to a great extent on work in the detection and interpretation of microwave spectra. The microwave spectra of $\mathrm{CH}_{3} \mathrm{CH}_{2} \mathrm{OH}$ were first recorded in Refs. 11-13 and the values of its rotational constants estimated in Refs. 12 and 13. The results of these papers were only partially confirmed later. ${ }^{14}$ However, it shown for the first time in a study of the microwave spectrum of $\mathrm{CH}_{3} \mathrm{CHDOH}$ (Ref. 15) that the trans-conformer of ethanol has a symmetry plane and, therefore, belongs to the $C_{S}$ point group. The equation $8 m_{H} r_{\perp}^{2}=I_{a}+I_{b}-I_{c}$ holds for it. The rotational constants and components of the dipole moment were calculated for the trans-conformer and two nonequivalent gauche-conformers of the $\mathrm{CH}_{3} \mathrm{CHDOH}$ molecule. The structural parameters of the ethanol trans-conformer were calculated using some of the structural parameters of the methanol molecule: ${ }^{16} l_{\mathrm{CH}}\left(\mathrm{CH}_{3}\right)=1.0936 \pm 0.0032 \AA$, $l_{\mathrm{OH}}=0.9451 \pm 0.0024 \AA, l_{\mathrm{CH}}\left(\mathrm{CH}_{2}\right)=1.0936 \pm 0.0050 \AA, l_{\mathrm{CC}}$ $=1.5297 \pm 0.0020 \AA, l_{\mathrm{CO}}=1.4247 \pm 0.0025 \AA, \delta_{\mathrm{HCH}}\left(\mathrm{CH}_{3}\right)=$ $108^{\circ} 38^{\prime} \pm 42^{\prime} l_{\mathrm{HCH}}\left(\mathrm{CH}_{2}\right)=1.09^{\circ} 5^{\prime} \pm 33^{\prime} \delta_{\mathrm{CCH}}\left(\mathrm{CH}_{2}\right)=110^{\circ} 18^{\prime}$ $\pm 42^{\prime} \delta_{\mathrm{COH}}=108^{\circ} 32^{\prime} \pm 29^{\prime}, \delta_{\mathrm{CCO}}\left(\mathrm{CH}_{2}\right)=107^{\circ} 20^{\prime} \pm 14^{\prime}$. The values of the angle $\delta_{\mathrm{CCO}}$ were found to differ substantially from the tetrahedral value, in accord with the data of Ref. 16.

Microwave spectra of a number of other isotopic analogs of ethanol $\left(\mathrm{CD}_{3} \mathrm{CH}_{2} \mathrm{OH}, \mathrm{CH}_{3} \mathrm{CD}_{2} \mathrm{OH}, \mathrm{CD}_{3} \mathrm{CD}_{2} \mathrm{OH}\right.$, $\left.\mathrm{CD}_{3} \mathrm{CD}_{2} \mathrm{OD}, \mathrm{CH}_{3} \mathrm{CH}_{2} \mathrm{O}^{18} \mathrm{H}, \mathrm{CH}_{3} \mathrm{C}^{13} \mathrm{H}_{2} \mathrm{OH}\right)$ have been recorded and interpreted. ${ }^{17-20}$ In the meantime, the situation with microwave spectra of the gauche-conformer of normal ethanol remains unclear, ${ }^{15}$ because b-type transitions were not found in the recorded spectra, while the $Q$-branch with c-type transitions lay far beyond the range of the spectral range that was studied. The authors of Ref. 15 emphasize the untenability of Ref. 21, where it was stated that based on a review of the data in Ref. 22, the stable trans- and gaucheconformers have been detected. Evidently, the first reliable experimental data on the microwave spectra of the gaucheconformer of ethanol have been obtained by Kakar and Seibt. ${ }^{23}$ For the rotational constants of the ground state they found $A^{ \pm}=34272.84 \mathrm{MHz}, B^{ \pm}=9188.03 \mathrm{MHz}$, and 
$C^{ \pm}=8099.86 \mathrm{MHz}$, and for those of the first excited torsional state, $A^{-}=35112.23 \mathrm{MHz}, B^{-}=9196.98 \mathrm{MHz}$, and $C=8103.78 \mathrm{MHz}$. The interaction of the internal rotations and the rotations of the ethanol molecule as a whole was found to be large. Approximate methods were used to obtain initial estimates of the internal rotation barrier $\left(420.8 \mathrm{~cm}^{-1}\right)$ and the difference in the energies of the symmetric and antisymmetric torsional state $\left(3.2 \mathrm{~cm}^{-1}\right)$ for the hydroxyl group of the gauche-conformer. Richard Quade has made a significant contribution to the interpretation of the microwave spectra of ethanol. With one-dimensional models ${ }^{24}$ for the interaction of the torsional motion and rotation of the molecule as a whole, ${ }^{25}$ he has found that the internal rotation barrier for the methyl group in the gauche-conformer $\left(1331 \mathrm{~cm}^{-1}\right)$ is almost $15 \%$ greater than that for the transconformer $\left(1158 \mathrm{~cm}^{-1}\right)$. A potential function for the internal rotation of the hydroxyl group of the form

$$
V(\alpha)=\frac{1}{2} \sum_{n=1,3} V_{n}(1-\cos n \alpha),
$$

has been found, where $V_{1}=57.0 \mathrm{~cm}^{-1}, V_{2}=0.8 \mathrm{~cm}^{-1}, V_{3}$ $=395.0 \mathrm{~cm}^{-1}$. The improved values for the rotational constants are: $A=34881.70 \mathrm{MHz}, \quad B=9350.65 \mathrm{MHz}$, $C=8135.20 \mathrm{MHz}$ for the trans-conformer and $A^{+}$ $=34015.81 \mathrm{MHz}, B^{+}=9188.64 \mathrm{MHz}, C^{+}=8099.98 \mathrm{MHz}$, $A^{-}=34331.86 \mathrm{MHz}, B^{-}=9194.42 \mathrm{MHz}, C=8100.85 \mathrm{MHz}$ for the gauche-conformer. The difference between the energies of the trans- and gauche-conformers was calculated for the first time $\left(41.2 \pm 5.0 \mathrm{~cm}^{-1}\right)$, with the trans-conformer being energetically preferable to the gauche-conformer. The small energy gap between the conformers has made it possible to use microwave spectra of ethanol in gas clouds near nascent stars to determine their temperatures. ${ }^{26-28}$ In fact, the intensity ratio of the lines associated with transitions from trans- and gauche-conformers in these microwave spectra is determined by the populations of the corresponding states, which, in turn, depend on the temperature of the nearest star. It was also noted that using a one dimensional model ${ }^{24}$ for analyzing the microwave spectra of ethanol does not yield the customarily good agreement between the series of theoretically calculated and experimentally observed frequencies of the lines and some spectral parameters of the ethanol molecule. It as shown that the interaction between the motion of the two tops is substantial, as is the interaction between the torsional motion of the hydroxyl group in the gaucheconformer with the zero-point vibrations of the molecule.

A later analysis ${ }^{29,30}$ of the microwave spectra of the $\mathrm{CDH}_{2} \mathrm{CH}_{2} \mathrm{OH}$ molecule, which can exist in several nonequivalent conformations associated with rotation of the partially deuterated methyl group, made it possible to determine the inertial coefficients $\mathrm{D}$ and $\mathrm{E}$ and the centrifugal tension coefficients $\Delta$ and $\Delta_{J K}$ and also revealed additional problems with the agreement between the theoretical and experimental results for states with $J \geq 3$. An analysis of the microwave spectra of ethanol in the spectral ranges where transitions between highly excited states determine the rotational and torsional states of the molecules ${ }^{28}$ revealed that the current theoretical methods for analyzing the torsional-rotational motion of molecules with a large reserve of kinetic energy do not provide an adequate representation of the experimental observations. ${ }^{31}$ This can be explained $^{31}$ by the absence of complete data on the potential surfaces for the interaction of nonequivalent internal tops, difficulties in the choice of an optimum coordinate system, and the inadequacy of the analytic representation for the angular momentum operator describing the internal rotation for large quantum numbers. As a result, studies of the microwave spectrum of ethanol are more or less empirical in character. ${ }^{28,32}$ The authors recognize that the molecular parameters found by minimizing the deviation between the theoretical predicted fit and the experimental line frequencies may be far from the actual characteristics of the molecules or even from widely accepted theoretical estimates of these parameters. An analysis of a large amount of new spectral data ${ }^{32}$ made it possible to improve some of the values of the parameters. The lower level of the gauche-conformer is split into symmetric $\left(e_{1}\right)$ and antisymmetric $\left(o_{1}\right)$ states, of which the former is lower by $\left(o_{1}\right)$ $3.2752201 \mathrm{~cm}^{-1}$. The ground state of the trans-conformer $\left(e_{0}\right)$, on the other hand, is $39.4925075 \mathrm{~cm}^{-1}$ lower than the symmetric state of the gauche-conformer. The energy of the first excited torsional state of the hydroxyl group in the ethanol trans-conformer $\left(o_{2}\right)$ is $202.6 \mathrm{~cm}^{-1}$, and the corresponding excited levels of the gauche-conformer, $e_{2}$ and $o_{3}$, are separated from $e_{1}$ and $o_{1}$ by 199.075 and $239.825 \mathrm{~cm}^{-1}$. No splitting owing to the torsional motion of the methyl group in low torsional-rotational states has been observed experimentally. Because of the symmetry of the methyl group, all its torsional states (as in the case of the methanol molecule) collapse into $A$ - and $E$-types.

The cluster equilibrium of ethanol molecules in the gaseous and liquid phases is also of research interest. Calculations using the quantum cluster equilibrium (QCE) theory ${ }^{33,34}$ have shown that cyclical clusters, among which the pentamer predominates, are predominant in the pure liquid over a wide range of temperatures. ${ }^{35}$ At the same time, for temperatures that are not too low, QCE predicts that the monomer is present. Later QCE calculations ${ }^{36}$ using density functional theory and the wider basis B3LYP/6-311 G(p, d) have not yielded the expected improved agreement between a number of calculated and experimental parameters of liquid ethanol. This suggests that QCE overestimates the fraction of large clusters in the liquid phase, which leads to an elevated density for the liquid. This also indicates that in pure liquid ethanol, smaller cyclical clusters (trimers and tetramers) should be present. The predominance of the latter was predicted in Ref. 37. The characteristic time for breaking of the hydrogen bond (400-800 fs $)^{38}$ is considerably shorter than the characteristic time for the subsequent recombination (10-20 ps), and this may be the reason for the lower probability of formation of larger cyclical clusters, for which the quantum-chemical calculations predict a larger binding energy. A similar recombination time has been found ${ }^{39}$ for the hydrogen bonds in $\mathrm{CH}_{3} \mathrm{CH}_{2} \mathrm{OD}$. With laser excitation of the O-D bond vibrations of the end acceptor molecules of the oligomers, and a study of their dissociation and recombination kinetics, these authors provide convincing arguments in favor of a step-by-step mechanism for breaking of the hydrogen bonds. In it, over a characteristic time of 2-3 ps the excitation energy of the vibrational state of the O-D bond is redistributed over intramolecular degrees of freedom, and transferred to solvent molecules; only afterward does it reach and destroy the hydrogen bonds of the associates. A study of 
the deactivation kinetics of the vibrational energy of the $\mathrm{C}-\mathrm{O}$ bond in methanol and ethanol molecules has shown that this process takes place in two steps. ${ }^{40}$ In the first, the energy of the $\mathrm{C}-\mathrm{O}$ vibrations is transferred to high torsional states of the hydroxyl group with a characteristic time that is the same for both molecules (3.2 ps). Then the energy of the intermediate state is transferred to lower-frequency modes. Here it turns out that the appearance of additional lowenergy degrees of freedom owing to the rotation of the methyl group in the ethanol molecule greatly reduces the characteristic time for this process $(12 \pm 2 \mathrm{ps})$ compared to that for methanol $(28 \pm 1 \mathrm{ps})$. The conformational sensitivity of photoelectron spectroscopy for the inner shells of the ethanol molecule has been demonstrated by removing the 1s-electrons from the carbon atoms in the methylene and methyl groups. ${ }^{41}$ It has been shown that, in the latter case, a torsional rotation is excited in the gauche-conformer and this leads to broadening of the low-frequency absorption band.

There have also been very extensive studies of the ethanol molecule by vibrational spectroscopy. The decreasing separation of the Raman scattering lines owing to valence vibrations of the hydroxyl group of the trans- $\left(3645 \mathrm{~cm}^{-1}\right)$ and gauche-conformers $\left(3635 \mathrm{~cm}^{-1}\right)$ (until they merge completely at $430 \mathrm{~K}$ ) has been attributed ${ }^{42}$ to a higher rate of inter-conformer transitions. IR studies of the mono- and poly-crystalline phases of ethanol ${ }^{43}$ show that the crystalline cell includes two chains of four molecules and has $C_{2 H}^{6}$ symmetry. Durig et al., ${ }^{44}$ have studied the IR spectra of polycrystalline ethanol, focussing on the interpretation of librational and translational modes. They have obtained IR spectra of ethanol in the gaseous phase. ${ }^{45}$ A qualitative interpretation of the spectrum has been proposed and the difference in the energies of the trans- and gauche-conformers $\left(110-179 \mathrm{~cm}^{-1}\right)$ has been estimated. A recent paper ${ }^{46}$ deals with determining the $r_{0}$ parameters for the geometry of the trans- and gauche-conformers of ethanol, determining the barriers of internal rotation, and the interpretation of the vibrational spectra. IR spectra $\left(300-4000 \mathrm{~cm}^{-1}\right)$ in the gaseous phase and IR spectra of solutions of ethanol in liquid xenon have been obtained for temperature ranging from -50 to $-100^{\circ} \mathrm{C}$. In addition extensive quantum chemical calculations have been carried out of the structure, force fields, and harmonic spectra of ethanol in the MP2/6-31G(d) (57 basis functions), MP2/acc-pVQZ (516 basis functions), and B3LYP/6-31G(d)-B3LYP/acc-pVQZ. Using the values of the rotational constants derived previously from the microwave spectra, for the trans- and gauche-conformers these authors have been able to calculate the $r_{0}$ parameters of the molecular structure by fitting nonempirical calculations of the parameters for optimal agreement between the experimental and calculated rotational constants. An interpretation of the vibrational spectra and of the potential energy distribution has been proposed using the symmetries as internal coordinates, although these are strictly applicable only for the gauche-conformer. Unfortunately, the force fields are not given in this paper and it is only pointed out that they are close for the trans- and gauche-conformers. In addition, the use of scaling factors can only distort the values of the force constants which determine the frequencies of the harmonic oscillations. A substantial amount of work on recording and interpreting the IR spectra of alcohols in matrix isolation has been done by Perchard et al. They may have given the first interpretation of the IR spectra of ethanol in 1968 (Ref. 47) and, based on recording and processing of the spectra of normal ethanol and its 11 isotopic analogs, Barnes and Hallam $^{48}$ proceeded to interpret the spectrum of ethanol in matrix isolation under the assumption that the transconformers predominate in the sample. Barnes also noted ${ }^{49}$ that the temperature dependence of the absorption bands of the hydroxyl group of ethanol in a nitrogen matrix may be caused by mutual gauche/trans conversion processes, but other spectral ranges were not examined. Perchard obtained IR spectra of ethanol in argon and nitrogen matrices. ${ }^{50}$ By calculating the structure and IR spectra of the two conformers in the B3LYP/6-311++G(2p, 2d) approximation and following the kinetics of the spectral transformations under laser irradiation of the sample at frequencies corresponding to the $\mathrm{O}-\mathrm{H}$ and $\mathrm{C}-\mathrm{O}$ bonds, he was able to come up with a reliable experimental attribution of the absorption bands in the vibrations of the gauche- and trans-conformers of ethanol. He also showed that an argon matrix stabilizes the transconformer, while both conformers are present in a nitrogen matrix and there are no inter-conformer transitions with laser irradiation in the ranges indicated here. It should, however, be noted that these calculations were done in the harmonic approximation and that the theoretical description of the normal vibrations is approximate.

\section{Experiment}

Argon (Elme Messer, $>99.995$ purity) was used to prepare the inert gas matrix. The liquid ethanol ( $>99.9$ purity) was initially purified further with removal of water molecules by keeping the sample in molecular sieves (3A) for $48 \mathrm{~h}$. Immediately prior to preparation of the mixture of gases for deposition of the matrix, the alcohol was degassed by multiple repetitions of a cycle of successive freezing, pumpdown, and unfreezing of the sample in a standard vacuum system. The samples for matrix isolation were fabricated by mixing ethanol vapor with argon under vacuum. The ratios of the numbers of alcohol molecules and argon atoms, 1:1000 and 1:2000, were determined using a standard manometer. The gas mixture was sprayed onto a substrate (CsI) cooled to $20 \mathrm{~K}$ in a closed-cycle helium cryostat (Leybold-Heraeus RW2). The gas flow in the cryostat was controlled by a needle valve. The rate of deposition was about 2 moles of gas mixture per hour, which ensures formation of a partially crystallized argon matrix. IR absorption spectra were recorded with a Bruker IFS 113 FTIR spectrometer. Spectral data were acquired in the range from 500 to $2000 \mathrm{~cm}^{-1}$ with a resolution of $0.5 \mathrm{~cm}^{-1}$. Each spectrum was recorded as the average of 128 scans in order to increase the signal-to-noise ratio. Spectra were taken at different temperatures from 20 to $45 \mathrm{~K}$ with a step size ranging from 1 to $5 \mathrm{~K}$.

\section{Computational technique}

The spectral-structural characteristics of the ethanol monomers were calculated using the GAUSSIAN 03 program package $^{51}$ in the B3LYP/cc - pVQZ approximation. B3LYP is a density functional theory (DFT) method for including exchange effects and electron correlation effects. It is currently the most widely tested and used hybrid method; it 
combines five functionals, of which three are exchange functionals (Becke + Slater $+\mathrm{HF})$ and two (LYP + VWN5) account for the electron correlation. ${ }^{52-54}$ The family of Dunning basis ${ }^{55}$ sets of general form cc-pVXZ, where $X=D$, $\mathrm{T}, \mathrm{Q}, 5,6$, etc., successively approaches the complete set of basis function and are also correlation consistent and valence split. Our earlier studies have shown that the combination B3LYP/cc-pVDZ already provides good reproduction of the frequencies of the harmonic vibrations of a number of organic molecules. ${ }^{56}$ Thus, our choice of this approximation (B3LYP/ cc-pVQZ) for the calculations was determined by the published data and by our earlier studies.

The internal energy is calculated as a function of internal rotation angle of the hydroxyl group with a step size of $20^{\circ}$, taking the energy of the zero-point vibrations into account in the harmonic approximation (ZPE). The dependence of the internal energy on the internal angle of rotation of the methyl group for the gauche- and trans-conformers of ethanol was calculated with a step size of $20^{\circ}$. The geometry of both conformers was optimized with respect to all the internal coordinates.

Figure 1 shows an ethanol molecule in the transconfiguration with the atoms numbered. The following natural coordinates were used to describe the vibrational motion of both of the ethanol conformers,

$$
\begin{gathered}
Q_{\mathrm{CO}}-l_{14} ; Q_{\mathrm{CC}}-l_{16} ; \varphi-\varphi_{213} ; \\
\alpha_{1}-\varphi_{214} ; \alpha_{2}-\varphi_{314} ; \beta_{1}-\varphi_{216} ; \quad \beta_{2}-\varphi_{316} ; \\
\gamma-\varphi_{416} ; \quad \theta-\varphi_{145} ; \delta_{1}-\varphi_{167} ; \delta_{2}-\varphi_{168} ; \delta_{3}-\varphi_{169} ; \\
\chi_{1}-\varphi_{768} ; \chi_{2}-\varphi_{769} ; \chi_{3}-\varphi_{869} ; \\
\tau_{1}-\varphi_{6145} ; \quad \tau_{2}-\varphi_{4167} .
\end{gathered}
$$

The force fields and frequencies of the harmonic vibrations were calculated for both ethanol conformers. It was found that most of the force constants in the trans- and gauche-conformers are essentially the same. The force constants associated with the changes in the length or deformation of the angle for the O-H group $(\theta)$ are an exception, as are the constants for the interactions of these coordinates with the other natural coordinates. The IR and Raman spectra were calculated in the anharmonic approximation for each of the optimized structures, with possible shifts in the vibrational frequencies owing to Fermi and Darling-Dennison resonances taken into account. An alternative way of accounting for the anharmonicity effects was carried out by replacing the true mass of the hydrogen atoms by the "spectroscopic" mass $(1.088 \mathrm{amu})$ in the configurations of the trans- and gaucheconformers of ethanol optimized in the B3LYP/cc-pVQZ approximation. Here the harmonic force fields calculated in the same approximation were used. The vibrational spectra of

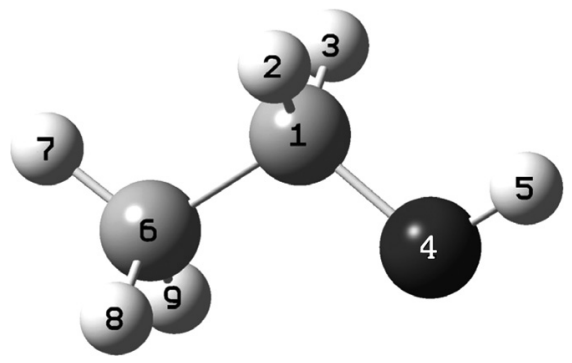

FIG. 1. The trans-conformer of the ethanol molecule with the atoms numbered. the trans- and gauche-conformers calculated in the harmonic and anharmonic approximations are shown in Table 1.

The cubic and quartic force constants in normal coordinates were calculated. Since the 21 normal vibrations of the trans-conformer break up into $13 A^{\prime}+8 A^{\prime \prime}$, all the cubic and quartic force constants, which contain derivatives of odd order with respect to normal coordinates of type $A^{\prime \prime}$, go to zero, since the potential energy must be invariant under the symmetry operations. In the case of the gauche-conformer, all the anharmonic force constants can be nonzero. The set of anharmonic force constants made it possible to construct ${ }^{57}$ a matrix of the anharmonicity constants $\chi_{i j} ; 1 \leq i \leq 21 ; 1 \leq j \leq i$. If the anharmonicity constants are given in inverse centimeters, then the vibrational frequency corrected for the anharmonicity ${ }^{58}$ is given by

$$
\mathrm{v}_{i}=\omega_{j}+2 \chi_{i i}+\frac{1}{2} \sum_{j \neq i} \chi_{i j} .
$$

The vibrational frequencies perturbed by the Fermi resonance were determined by diagonalizing a matrix whose diagonal elements are the fundamental vibrations, overtones, and composite anharmonic frequencies unperturbed by the Fermi resonance, while the nondiagonal elements are the corresponding cubic force constants expressed in inverse centimeters. ${ }^{57,59}$ According to the calculations, the Fermi resonances cause only a slight shift in the frequencies of some of the fundamental vibrations in the trans-conformer, and the composite vibrations and overtones can be omitted from the analysis, since they receive no more than $2.5 \%$ of the intensity of the low intensity fundamental vibrations (Table 1). At the same time, in the gauche-conformer the calculations show that the composite vibration at $1036.1 \mathrm{~cm}^{-1}$ composed of torsional vibrations of the methyl group and pendulum vibrations of $\mathrm{C}-\mathrm{H}$ bonds in methylene and methyl groups takes up $39.4 \%$ of the intensity of the fundamental $1051.3 \mathrm{~cm}^{-1}$ vibration, which is one of the most intense in the IR spectrum of the gauche-conformer. Thus, because of the Fermi resonance the composite $1036.1 \mathrm{~cm}^{-1}$ vibration has an intensity comparable to the intensity of the fundamental vibrations and can show up in the sample IR spectra.

\section{Discussion of results}

\subsection{Structure of the trans- and gauche-conformers of ethyl alcohol}

Table 2 lists the energies of the ground state vibrations and the heights of the barriers for the trans- and gaucheconforms of ethanol, with and without the ZPE, in the harmonic and anharmonic approximations for the rotation of the hydroxyl and methyl tops.

According to the data obtained in the B3LYP/cc-pVQZ approximation, in the harmonic approximation the transconformer ZPE is roughly $5.33 \mathrm{~cm}^{-1}$ greater than for the gauche-conformer. In the anharmonic approximation, the ZPE of the gauche-conformer is $5.47 \mathrm{~cm}^{-1}$ greater than that of the trans-conformer. Thus, with the correction for the zero-point energy in the anharmonic approximation the calculated difference between the gauche- and trans-conformers $\left(+35.38 \mathrm{~cm}^{-1}\right)$ is in very good agreement with experiment $\left(+39.49 \mathrm{~cm}^{-1}\right)$. On the whole, the calculation correctly reflects the relationship 
TABLE 1. Calculated vibrational frequencies in the harmonic and anharmonic approximations for the trans- and gauche-conformers of the ethanol molecule.

\begin{tabular}{|c|c|c|c|c|c|c|}
\hline $\begin{array}{l}\text { No., } \\
\text { symmetry }\end{array}$ & $\begin{array}{c}\tilde{\nu} \text { (harmonic) } \\
\mathrm{cm}^{-1}\end{array}$ & $\begin{array}{c}\tilde{\nu} \text { (anharmonic) } \\
\mathrm{cm}^{-1}\end{array}$ & $\begin{array}{l}\text { Levels (neglecting } \\
\text { Fermi resonances) }\end{array}$ & $\begin{array}{l}\text { Spectroscopic } \\
\text { mass }\end{array}$ & $I, \mathrm{~km} / \mathrm{mole}$ & Distribution of vibrational potential energy \\
\hline \multicolumn{7}{|c|}{ Trans-conformer } \\
\hline 1. $A^{\prime}$ & 235.1 & 215.1 & & 207.3 & 45.9 & $40.7 \% \tau_{1}+52.5 \% \tau_{2}$ \\
\hline 2. $A^{\prime}$ & 279.4 & 200.1 & & 256.4 & 70.0 & $62.2 \% \tau_{1}+22.3 \% \tau_{2}$ \\
\hline 3. $A^{\prime}$ & 418.9 & 414.1 & & 408.6 & 10.6 & $\begin{array}{l}27.1 \% \gamma+7.9 \% \theta+8 \% \delta_{1}+8 \% \delta_{2}+8.4 \% \alpha_{1} \\
+8.4 \% \alpha_{2}+4.4 \% \varphi+4.4 \% \beta_{1}+4.4 \% \beta_{2}\end{array}$ \\
\hline 4. $A^{\prime \prime}$ & 823.4 & 815.1 & & 785.9 & 0.11 & $\begin{array}{l}17.9 \% \delta_{2}+17.9 \% \delta_{3}+16.9 \% \beta_{1}+16.9 \% \beta_{2}+16.9 \% \beta_{2} \\
\quad+12.1 \% \tau_{2}+3.8 \% \chi_{1}+3.8 \chi_{2}+3.7 \% \alpha_{1}+3.7 \% \alpha_{2}\end{array}$ \\
\hline 5. $A^{\prime}$ & 897.9 & 879.9 & & 880.4 & 12.1 & $\begin{array}{c}20.0 \% \delta_{1}+17.5 \% \mathrm{Q}_{\mathrm{CO}}+16.3 \% \mathrm{Q}_{\mathrm{CC}}+8.6 \% \delta_{2} \\
+8.6 \% \delta_{3}+4.9 \% \beta_{1}+4.9 \% \beta_{2}+4.6 \% \chi_{3}\end{array}$ \\
\hline 6. $A^{\prime}$ & 1030.6 & 1000.9 & & 1011.6 & 64.6 & $\begin{array}{c}21.5 \% Q_{\mathrm{CC}}+17.5 \% \theta+11.6 \% Q_{\mathrm{CO}}+10.6 \% \delta_{1} \\
+7.3 \% \delta_{2}+7.3 \% \delta_{3}+4.4 \% \alpha_{1}+4.4 \% \alpha_{2}+4.4 \% \gamma\end{array}$ \\
\hline 7. $A^{\prime}$ & 1101.9 & 1078.5 & & 1075.2 & 24.3 & $\begin{array}{c}19.9 \% Q_{\mathrm{CO}}+17.8 \% \delta_{1}+8.4 \% \delta_{2}+8.4 \% \delta_{3}+7.3 \% \theta+ \\
6.5 \% \gamma+4.5 \% Q_{\mathrm{CC}}+3.6 \% \alpha_{1}+3.6 \% \alpha_{2}+2.6 \% \chi_{1}\end{array}$ \\
\hline $8 . A^{\prime \prime}$ & 1181.6 & 1152.8 & 1149.8 & 1152.9 & 3.7 & $\begin{array}{l}18.5 \% \alpha_{1}+18.5 \% \alpha_{2}+14.2 \% \delta_{2}+14.2 \% \delta_{3}+6.5 \% \chi_{1} \\
+6.5 \% \chi_{2}+5.7 \% \beta_{1}+5.7 \% \beta_{2}+4.7 \% \tau_{2}+3.8 \% \tau_{1}\end{array}$ \\
\hline 9. $A^{\prime}$ & 1268.9 & 1229.5 & & 1231.4 & 70.9 & $\begin{array}{l}26.9 \% \theta+8.9 \% \beta_{1}+8.9 \% \beta_{2}+7.8 \% \delta_{1}+5.9 \% \delta_{2}+5.9 \% \delta_{3} \\
\quad+5.6 \% \alpha_{1}+5.6 \% \alpha_{2}+4.7 \% Q_{\mathrm{CO}}+4.1 \% \chi_{1}+4.1 \% \chi_{2}\end{array}$ \\
\hline 10. $A^{\prime \prime}$ & 1302.0 & 1267.9 & 1267.9 & 1257.0 & 0.01 & $\begin{array}{l}17.1 \% \beta_{1}+17.1 \% \beta_{1}+14.3 \alpha_{1}+14.3 \% \alpha_{2}+8.9 \% \delta_{2} \\
+8.9 \% \delta_{3}+4.2 \% \chi_{1}+4.2 \% \chi_{2}+6.2 \% \tau_{2}+3.0 \% \tau_{1}\end{array}$ \\
\hline 11. $A^{\prime}$ & 1408.3 & 1373.4 & & 1362.3 & 2.2 & $\begin{array}{c}15.4 \% \delta_{1}+15.0 \% \chi_{1}+15.0 \% \chi_{2}+12.9 \% \delta_{2}+12.9 \% \delta_{3}+1.4 \% \\
\chi_{3}+4.7 \% \beta_{1}+4.7 \% \beta_{2}+4.5 \% \alpha_{1}+4.5 \% \alpha_{2}\end{array}$ \\
\hline 12. $A^{\prime}$ & 1454.1 & 1446.9 & 1449.9 & 1416.9 & 11.2 & $\begin{array}{l}12.3 \% \beta_{1}+12.3 \% \beta_{2}+12.1 \% \alpha_{1}+12.1 \% \alpha_{2}+12.0 \% \theta \\
+8.3 \% Q_{\mathrm{CC}}+6.9 \% \chi_{1}+6.9 \% \chi_{2}+4.1 \% \delta_{2}+4.1 \% \delta_{3}\end{array}$ \\
\hline 13. $A^{\prime}$ & 1483.8 & 1459.6 & & 1427.3 & 5.7 & $28.9 \% \chi_{1}+28.9 \% \chi_{2}+21.1 \% \tau_{2}+8.2 \% \delta_{2}+8.2 \% \delta_{3}$ \\
\hline 14. $A^{\prime}$ & 1500.9 & 1484.1 & & 1445.2 & 2.64 & $\begin{aligned} 29.3 \% \chi_{3}+ & 12.2 \% \chi_{1}+12.2 \% \chi_{1}+9.7 \% \varphi+8.8 \% \delta_{2} \\
& +8.8 \% \delta_{3}+4.8 \% \beta_{1}+4.8 \% \beta_{2}\end{aligned}$ \\
\hline 15. $A^{\prime}$ & 1529.6 & 1475.1 & & 1475.9 & 1.24 & $\begin{array}{c}32.4 \% \varphi+10.2 \% \chi_{3}+9.5 \% \alpha_{1}+9.5 \% \alpha_{2}+5.9 \% \beta_{1} \\
+5.9 \% \beta_{2}+4.1 \% \chi_{1}+4.1 \% \chi_{2}+3.5 \% \delta_{1}\end{array}$ \\
\hline \multicolumn{7}{|c|}{ Gauche-conformer } \\
\hline 1. & 257.6 & 233.6 & & 234.8 & 8.4 & $23.8 \% \tau_{1}+60.6 \% \tau_{2}$ \\
\hline 2. & 272.2 & 212.5 & & 259.9 & 104.4 & $65.2 \% \tau_{1}+13.9 \% \tau_{2}$ \\
\hline 3. & 420.9 & 415.5 & & 412.5 & 11.9 & $\begin{array}{l}21.3 \% \gamma+20.9 \% \tau_{1}+11.8 \% \delta_{1}+7.6 \% \beta_{1}+7.2 \% \delta_{3} \\
+5.9 \% \beta_{2}+5.5 \% \delta_{2}+3.8 \% Q_{\mathrm{CC}}+3.8 \% \alpha_{1}+3.6 \% \alpha_{2}\end{array}$ \\
\hline 4. & 806.5 & 804.2 & & 775.9 & 2.9 & $\begin{array}{c}17.2 \% \delta_{2}+16.8 \% \delta_{3}+16.7 \% \beta_{2}+15.8 \% \beta_{1} \\
+11.9 \% \tau_{2}+4.5 \% \alpha_{2}+4.0 \% \alpha_{1}+3.7 \% \chi_{1}+3.4 \% \chi_{2}\end{array}$ \\
\hline 5. & 883.2 & 866.6 & & 868.4 & 11.1 & $\begin{array}{c}20.2 \% \delta_{1}+18.6 \% Q_{\mathrm{CC}}+15.8 \% Q_{\mathrm{CO}}+9.8 \% \delta_{3} \\
+7.2 \% \delta_{2}+5.1 \% \beta_{1}+4.6 \% \chi_{3}+4.0 \% \alpha_{1}+3.1 \% \beta_{2}\end{array}$ \\
\hline 6. & 1058.9 & 1035.0 & & 1033.8 & 6.7 & $\begin{array}{c}21.6 \% Q_{\mathrm{CC}}+12.7 \% \delta_{2}+12.6 \% Q_{\mathrm{CO}}+11.7 \% \delta_{1} \\
+10.0 \% \theta+9.5 \% \alpha_{2}+5.1 \% \gamma+4.7 \% \chi_{2}+3.3 \% \delta_{3}\end{array}$ \\
\hline 7. & 1069.2 & 1051.3 & 1045.3 & 1057.3 & 73.2 & $\begin{array}{l}18.3 \% \theta+16.3 \% Q_{\mathrm{CO}}+11.9 \% \delta_{2}+10.3 \% \delta_{1} \\
+10.2 \% \alpha_{2}+7.1 \% \alpha_{1}+6.8 \% Q_{\mathrm{CC}}+3.3 \% \chi_{2}\end{array}$ \\
\hline $18+21$ & & 1036.1 & 1042.1 & & 46.9 & $\left(\delta_{\text {rock }}^{\mathrm{CH}_{2}}+\delta_{\text {rock }}^{\mathrm{CH}_{3}}\right)+\left(23.8 \% \tau_{1}+60.6 \% \tau_{2}\right)$ \\
\hline 8. & 1134.5 & 1114.7 & 1111.6 & 1112.5 & 4.3 & $\begin{array}{c}16.2 \% \delta_{3}+13.6 \% \delta_{1}+12.9 \% \alpha_{1}+9.4 \% Q_{\mathrm{CO}} \\
+8.1 \% \alpha_{2}+7.1 \% \gamma+6.1 \% \theta+6.1 \% \chi_{1}+6.0 \% \tau_{1}\end{array}$ \\
\hline $17+21$ & & 1120.4 & 1117.7 & & 0.6 & \\
\hline 9. & 1281.3 & 1247.8 & & 1246.0 & 11.8 & $16.6 \% \beta_{2}+13.3 \% \theta+12.3 \% \beta_{1}+10.8 \% \delta_{3}+8.5 \% \alpha_{2}$ \\
\hline $14+20$ & & 1320.0 & 1322.7 & & 0.8 & \\
\hline 10. & 1373.7 & 1328.9 & & 1335.9 & 4.4 & $\begin{array}{l}24.0 \% \alpha_{1}+20.5 \% \theta+18.3 \% \beta_{1}+7.5 \% \chi_{3}+5.8 \% \delta_{1} \\
+3.3 \% \alpha_{2}+3.2 \% \tau_{1}+2.6 \% Q_{\mathrm{CO}}+2.4 \% \chi_{1}+2.2 \% \beta_{2}\end{array}$ \\
\hline 11. & 1404.1 & 1376.3 & 1375.2 & 1361.6 & 4.8 & $\begin{array}{c}16.0 \% \chi_{2}+13.4 \% \delta_{1}+12.1 \% \delta_{3}+11.3 \% \chi_{3} \\
+11.0 \% \delta_{2}+8.8 \% \chi_{1}+7.0 \% \alpha_{2}+6.3 \% \theta+3.7 \% \beta_{2}\end{array}$ \\
\hline 12. & 1418.3 & 1389.9 & 1388.3 & 1380.7 & 40.6 & $\begin{array}{l}15.0 \% \alpha_{2}+12.7 \% \beta_{2}+10.2 \% \beta_{1}+9.6 \% \chi_{1}+7.8 \% \theta \\
+8.3 \% \alpha_{1}+7.1 \% Q_{\mathrm{CC}}+6.9 \% \chi_{2}+6.3 \% \delta_{2}+6.3 \% \delta_{3}\end{array}$ \\
\hline $15+19$ & & 1453.8 & 1455.6 & & 0.5 & \\
\hline 13. & 1488.5 & 1469.5 & 1468.5 & 1435.3 & 6.7 & $27.5 \% \chi_{1}+26.7 \% \chi_{2}+20.0 \% \tau_{2}+8.7 \% \delta_{2}+6.7 \% \delta_{3}$ \\
\hline 14. & 1493.8 & 1476.9 & 1475.5 & 143.8 .9 & 1.7 & $\begin{array}{c}27.6 \% \chi_{3}+18.6 \% \varphi+9.5 \% \chi_{1}+8.6 \% \chi_{2}+8.6 \% \delta_{1} \\
\quad+7.7 \% \beta_{1}+6.9 \% \delta_{3}+3.3 \% \beta_{2}+3.2 \% \delta_{2}\end{array}$ \\
\hline 15. & 1518.4 & 1477.0 & & 1468.4 & 0.7 & $\begin{array}{c}30.9 \% \varphi+13.5 \% \chi_{3}+9.7 \% \alpha_{2}+9.6 \% \alpha_{1}+9.5 \% \beta_{1} \\
+7.9 \% \beta_{2}+6.7 \% \chi_{2}+5.8 \% \chi_{1}+5.2 \% \delta_{1}\end{array}$ \\
\hline
\end{tabular}

between the internal rotation barriers (Table 2), but in the case of the hydroxyl group the barriers are somewhat excessive and in the case of the methyl group, somewhat low. Some structural parameters of the ethanol molecule (rotational constants $A, B$, and $C$, and the centrifugal tension constants $\Delta_{J}, \Delta_{J K}, \Delta_{K}$, $\left.\delta_{j}, \delta_{k}\right)$ are listed in Table 3. 
TABLE 2. Relative energies of the ground states and barriers to internal rotation of the hydroxyl and methyl groups of trans- and gauche-conformers of the ethanol molecule.

\begin{tabular}{|c|c|c|c|c|c|c|}
\hline \multirow[b]{2}{*}{ Computational technique } & \multicolumn{2}{|c|}{ Energy of ground states, $\mathrm{cm}^{-1}$} & \multicolumn{2}{|c|}{ Barriers to rotation of hydroxyl group, $\mathrm{cm}^{-1}$} & \multicolumn{2}{|c|}{ Barriers to rotation of methyl group, $\mathrm{cm}^{-}$} \\
\hline & Trans & Gauche & Trans & G-T/G-T & Trans & Gauche \\
\hline Without ZPE & 0 & +29.91 & 357.6 & $365.6 / 422.0$ & 1096.72 & 1163.23 \\
\hline ZPE (harm.) & 0 & +24.58 & 357.6 & $362.8 / 421.4$ & & \\
\hline ZPE (anharm.) & 0 & +35.38 & 338.8 & $365.6 / 422.0$ & & \\
\hline Exp. (Ref. 32) & 0 & +34.49 & 391.1 & $347.4 / 371.7$ & 1173.8 & 1331.0 \\
\hline
\end{tabular}

TABLE 3. Some structural parameters and the centrifugal tension constants of the trans- and gauche-conformers of the ethanol molecule.

\begin{tabular}{lcccc}
\hline \hline Structural constants & Trans- (calculated), $\mathrm{cm}^{-1}$ & Trans- (experiment), $\mathrm{cm}^{-1}$ & Gauche- (calculated), $\mathrm{cm}^{-1}$ & Gauche- (experiment), cm $^{-1}$ \\
\hline$A$ & 1.17639 & 1.16386 (Ref. 25) & 1.15499 & 1.13912 (Ref. 25) \\
$B$ & 0.31217 & 0.3104 (Ref. 25) & 0.30548 & 0.30655 (Ref. 25) \\
$C$ & 0.27177 & 0.27136 (Ref. 25) & 0.27019 & 0.27019 (Ref. 25) \\
$\Delta_{J}$ & $8220.23 \mathrm{~Hz}$ & $8537.36 \mathrm{~Hz}$ (Ref. 60) & $8935.86 \mathrm{~Hz}$ & $8870.76 \mathrm{~Hz}($ Ref. 28) \\
$\Delta_{J K}$ & $-19758.362 \mathrm{~Hz}$ & $-28778.5 \mathrm{~Hz}$ (Ref. 60) & $-35224.5 \mathrm{~Hz}$ & $-18735.4 \mathrm{~Hz}$ (Ref. 28) \\
$\Delta_{K}$ & $22307.198 \mathrm{~Hz}$ & $25246.0 \mathrm{~Hz}$ (Ref. 60) & $25163.1 \mathrm{~Hz}$ & $29150.0 \mathrm{~Hz}($ Ref. 28) \\
$\delta_{i}$ & $1569.72 \mathrm{~Hz}$ & $1737.66 \mathrm{~Hz}$ (Ref. 60) & $1786.42 \mathrm{~Hz}$ & $13451.6 \mathrm{~Hz}$ \\
$\delta_{k}$ & $9048.56 \mathrm{~Hz}$ & $6681.4 \mathrm{~Hz}$ (Ref. 60) & \\
\hline
\end{tabular}

It should be noted that the calculations are basically in very good agreement with experimental data according to the spectral constants which determine the characteristics of the microwave spectra. Some of the calculated and experimental parameters of the ethanol molecule are listed in Table 4.

It can be seen in Table 4 that the B3LYP/cc-pVQZ approximation reproduces the length of the chemical bonds and the valence angles with high accuracy. This is to be expected, given the very good agreement of the data in Table 3 . The difference between the calculated and experimental values of the $\mathrm{CCOH}$ dihedral angle is, however, substantial.

\subsection{FTIR spectra of ethyl alcohol in an argon matrix}

As noted above, the absorption spectra of ethanol in argon matrices were recorded for concentration ratios of
1:1000 and 1:2000. A comparison of these spectra can, on one hand, reveal the bands owing to the formation of associates, which is more probable for the first sample, and, on the other, the bands owing to buildup of impurities, which are more probable for the second sample because the substance has been condensed for a longer time on the window. ${ }^{61}$ The absorption bands owing to valence vibrations of $\mathrm{C}-\mathrm{H}$ bonds are identified in Ref. 62. The spectra taken over the interval $600-1500 \mathrm{~cm}^{-1}$ are shown in Fig. 2.

On comparing the IR absorption spectra with different ethanol concentrations, we were able to assign the bands at 722,733 , and $1473 \mathrm{~cm}^{-1}$ in the spectrum with a concentration ratio of 1:2000 to impurities in the sample. In addition, $\mathrm{CO}_{2}$ is present as an impurity in both samples and the absorption bands at $662,663.5,2339.5$, and $2345 \mathrm{~cm}^{-1}$ were

TABLE 4. Geometric parameters of trans- $(t)$ and gauche- $(g)$ conformers of the ethanol molecule.

\begin{tabular}{|c|c|c|c|c|c|c|c|c|}
\hline Parameter & Calculated & Exp. (Ref. 46) & Parameter & Calculated & Exp. (Ref. 46) & Parameter & Calculated & Exp. (Ref. 46) \\
\hline$r_{\mathrm{O}-\mathrm{H}}, \AA$ & $\frac{0.961(t)}{0.962(g)}$ & $\frac{0.962(t)}{0.963(g)}$ & $\delta_{\mathrm{COH}}, \operatorname{deg}$ & $\frac{108.75(t)}{108.40(g)}$ & $\frac{107.5(t)}{107.0(g)}$ & $\delta_{\mathrm{HCH}}^{\mathrm{CH}_{3}}, \operatorname{deg}$ & $\begin{array}{l}\frac{108.50(t)}{108.30(t)} \\
108.17(g) \\
108.52(g) \\
107.63(g)\end{array}$ & $\begin{array}{l}\frac{108.8(t)}{108.8(t)} \\
108.4(g) \\
108.8(g) \\
107.9(g)\end{array}$ \\
\hline$r_{\mathrm{O}-\mathrm{C}}, \AA$ & $\frac{1.427(t)}{1.425(g)}$ & $\frac{1.428(t)}{1.430(g)}$ & $\delta_{\mathrm{CCO}}, \operatorname{deg}$ & $\frac{108.00(t)}{112.93(g)}$ & $\frac{107.6(t)}{112.5(g)}$ & $\delta_{\mathrm{CCH}}^{\mathrm{CH}_{3}}, \operatorname{deg}$ & $\begin{array}{c}\frac{110.54(t)}{110.48(t)} \\
111.041(\mathrm{~g}) \\
110.58(\mathrm{~g}) \\
110.78(\mathrm{~g})\end{array}$ & $\begin{array}{l}\frac{110.7(t)}{110.7(t)} \\
110.8(g) \\
110.2(g) \\
110.7(g)\end{array}$ \\
\hline$r_{\mathrm{C}-\mathrm{C}}, \AA$ & $\frac{1.515(t)}{1.521(g)}$ & $\frac{1.512(t)}{1.523(g)}$ & $\delta_{\mathrm{HCH}}^{\mathrm{CH}_{2}}$, deg & $\frac{107.56(t)}{107.18(g)}$ & $\frac{108.5(t)}{107.7(g)}$ & $\tau_{\mathrm{CCOH}}$, deg & $\frac{180.00(t)}{-61.67(g)}$ & $\frac{180.0(t)}{-56.9(g)}$ \\
\hline$r_{\mathrm{C}-\mathrm{H}}^{\mathrm{CH}_{2}}, \AA$ & $\begin{array}{l}1.097(\mathrm{t}) \\
1.090(\mathrm{~g}) \\
1.097(\mathrm{~g})\end{array}$ & $\begin{array}{l}1.099(t) \\
1.093(g) \\
1.100(g)\end{array}$ & $\delta_{\mathrm{CCH}}^{\mathrm{CH}_{2}}$, deg & $\begin{array}{l}110.01(t) \\
110.26(\mathrm{~g}) \\
110.26(\mathrm{~g})\end{array}$ & $\begin{array}{l}109.6(\mathrm{t}) \\
110.2(\mathrm{~g}) \\
110.5(\mathrm{~g})\end{array}$ & & & \\
\hline$r_{\mathrm{C}-\mathrm{H}}^{\mathrm{CH}_{3}}, \AA$ & $\begin{array}{l}\frac{1.091(t)}{1.090(t)} \\
1.092(\mathrm{~g}) \\
1.093(\mathrm{~g}) \\
1.090(\mathrm{~g})\end{array}$ & $\begin{array}{l}\frac{1.095(t)}{1.094(t)} \\
1.096(\mathrm{~g}) \\
1.097(\mathrm{~g}) \\
1.094(\mathrm{~g})\end{array}$ & $\delta_{\mathrm{OCH}}^{\mathrm{CH}_{2}}$, deg & $\begin{array}{l}110.63(\mathrm{t}) \\
110.70(\mathrm{~g}) \\
105.20(\mathrm{~g})\end{array}$ & $\begin{array}{l}109.8(\mathrm{t}) \\
110.4(\mathrm{~g}) \\
105.2(\mathrm{~g})\end{array}$ & & & \\
\hline
\end{tabular}




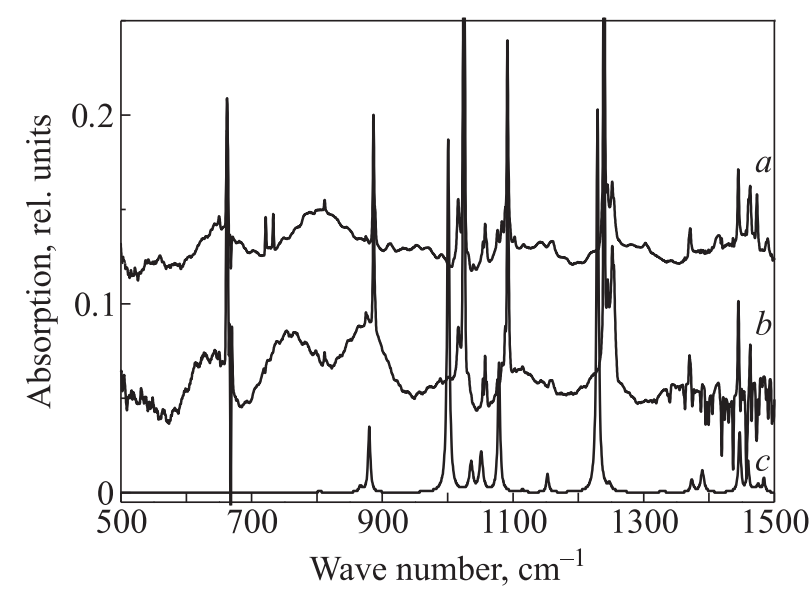

FIG. 2. FTIR spectra of the ethanol molecule in the range $600-1500 \mathrm{~cm}^{-1}$, with experimentally recorded spectra for molecular ratios of 1:2000 (a) and 1:1000 (b). The theoretically calculated spectrum is shown as curve c. attributed to it. The peak wave numbers and relative intensities of the bands are listed in Table 5 .

The existence of IR and Raman scattering spectra of ethanol in matrix isolation, in a xenon solution, and in the gaseous phase is extremely important for the attribution of bands to vibrations of the molecule. As noted above, this makes it possible to reveal bands caused by impurities and aggregate formation. In addition, the multiplet structure of several of the absorption bands in argon and nitrogen matrices is often caused by a "site" effect. This effect does not, as a rule, show up in supercooled solutions of the inert gases. ${ }^{61}$ Since the theoretical calculations have been done for isolated molecules, it is correct to compare the results with the frequencies for the gaseous phase. Shifts in the vibrational frequencies in matrices relative to those observed in the gaseous phase are attributed to matrix effects, ${ }^{63}$ which are of the same nature as the frequency shifts of molecules in

TABLE 5. Frequencies and intensities of the experimentally observed absorption bands and scattering lines in IR and Raman scattering spectra of ethanol from the present work and published data.

\begin{tabular}{|c|c|c|c|c|c|c|c|c|c|c|}
\hline$\tilde{v}, \mathrm{~cm}^{-1}$ & $\begin{array}{l}I, \text { rel. } \\
\text { units }\end{array}$ & $\begin{array}{l}\text { Trans Ar } \\
\text { (Ref. 50) }\end{array}$ & $\begin{array}{l}\text { Trans } \mathrm{N}_{2} \\
\text { (Ref. 50) }\end{array}$ & $\begin{array}{l}\text { Trans Xe } \\
\text { (Ref. 46) }\end{array}$ & $\begin{array}{c}\text { Trans IR, } \\
\text { gas (Ref. 46) }\end{array}$ & $\begin{array}{l}\text { Trans Raman, } \\
\text { gas (Ref. 46) }\end{array}$ & $\begin{array}{c}\text { Gauche } \mathrm{N}_{2} \\
\text { (Ref. 50) }\end{array}$ & $\begin{array}{l}\text { Gauche } \\
\text { Xe [46] }\end{array}$ & $\begin{array}{c}\text { Gauche IR, } \\
\text { gas (Ref. 46) }\end{array}$ & $\begin{array}{c}\text { Gauche Raman } \\
\text { gas (Ref. 46) }\end{array}$ \\
\hline & & & & & 202.6 & & & & 195.8 & \\
\hline & & & & & & & & & 243.1 & \\
\hline & & & & & 244.4 & & & & 274.0 & \\
\hline & & 416.5 & & 419.0 & 418.0 & 422.0 & & 419.0 & 420.0 & 422.0 \\
\hline 651.0 & 0.01 & & & & & & & & & \\
\hline \multirow[t]{2}{*}{812.0} & 0.005 & 811.8 & & 797.0 & 801.0 & & 811.3 & 800.0 & 803.0 & \\
\hline & & & & & & & 812.1 & & & \\
\hline 886.5 & 0.1 & 886.4 & 887.6 & 887.0 & 892.0 & 883.0 & 884.6 & 879.0 & 879.0 & 883.0 \\
\hline 888.0 & 0.02 & 889.4 & & & & & 885.2 & & & \\
\hline 1016.0 & 0.03 & 1016.4102 & 1027.7 & 1025.0 & 1027.0 & 1026.0 & & & & \\
\hline 1025.6 & 0.6 & 5.0 & & & & & & & & \\
\hline 1054.0 & 0.01 & & & & & & & 1055.0 & 1058.0 & 1055.0 \\
\hline 1057.5 & 0.03 & & & & & & 1058.1 & & & \\
\hline \multirow[t]{2}{*}{1060.0} & 0.01 & & & & & & 1063.0 & & 1066.0 & \\
\hline & & & & & & & 1064.4 & & & \\
\hline 1076.6108 & 0.02 & 1076.9108 & & & & & & & & \\
\hline 3.0 & 0.02 & 3.5 & & & & & & & & \\
\hline 1088.0 & 0.01 & 3.5 & & & & & & & & \\
\hline 1092.0 & 0.2 & 1091.7 & 1090.7 & 1086.0 & 1090.0 & 1093.0 & & & & \\
\hline 1115.0 & 0.003 & & & & & & 1125.8 & 1116.0 & 1117.0 & 1117.0 \\
\hline 1145.0 & 0.005 & & 1139.9 & & & & & & & \\
\hline 1161.0 & 0.01 & 1161.0 & 1159.6 & 1156.0 & 1166.0 & & & & & \\
\hline 1239.0 & 0.6 & 1239.5 & 1256.3 & 1238.0 & 1241.0 & 1245.0 & & & & \\
\hline 1244.0 & 0.02 & & & & & & & 1248.0 & 1249.0 & 1245.0 \\
\hline 1252.0 & 0.02 & & & & & & & & & \\
\hline \multirow[t]{2}{*}{1252.0} & 0.02 & & 1274.8 & 1272.0 & 1275.0 & & 1260.6 & & & \\
\hline & & & & & & & 1337.3 & 1339.0 & 1342.0 & 1340.0 \\
\hline 1369.0 & 0.02 & & & & & & & & & \\
\hline \multirow[t]{2}{*}{1372.0} & 0.02 & 1371.5 & 1371.4 & 1367.0 & 1367.0 & & 1372.2 & 1373.0 & 1373.0 & 1370.0 \\
\hline & & & & & & & 1389.1 & 1388.0 & 1394.0 & 1395.0 \\
\hline 1412.0 & 0.01 & & 1411.7 & & & & & & & \\
\hline 1415.0 & 0.01 & 1416.3 & & 1446.0 & 1450.0 & 1430.0 & & & & \\
\hline 1444.7 & 0.03 & 1445 & 1445.8 & 1453.0 & 1455.0 & & & & & \\
\hline \multirow[t]{2}{*}{1461.0} & 0.02 & & 1463.5 & & & & 1451.8 & 1460.0 & 1460.0 & 1460.0 \\
\hline & & & & & & & 1456.1 & & & \\
\hline 1462.7 & 0.02 & 1463.3 & & & & & 1456.0 & 1464.0 & 1465.0 & \\
\hline 1487.5 & 0.015 & 1487.0 & & 1483.0 & 1480.0 & 1460.0 & 1485.3 & & & \\
\hline 1490.0 & 0.02 & & 1490.2 & 1501.0 & 1500.0 & & & 1492.0 & 1493.0 & \\
\hline
\end{tabular}


nonpolar solvents. It should also be recalled that both conformers are present in the gaseous phase at room temperature, with more than $60 \%$ of the molecules as gaucheconformers owing to configurational degeneracy. ${ }^{46}$ A number of absorption bands of ethanol in $\mathrm{Ar}$ and $\mathrm{N}_{2}(1: 1800)$ at $9 \mathrm{~K}$ have a multiplet structure owing to the "site" effect. ${ }^{50}$ The spectra obtained here have a similar multiplet structure for both concentrations (1:1000 and 1:2000) at $20 \mathrm{~K}$. The lack of a concentration dependence for the intensity of the components is typical of the "site" effect, 63 and this proposition is also supported by the absence of a multiplet structure in the corresponding bands in a low-temperature spectrum of ethanol in Xe. ${ }^{46}$ The components related to this effect are placed in a single cell of Table 5. However, an argon matrix stabilizes the trans-conformer, ${ }^{50}$ while $\mathrm{N}_{2}$ allows both conformers to be present. In the latter case it has been shown experimentally $^{50}$ that reversible interconversion of gaucheand trans-conformers can take place during laser irradiation at the frequencies of the valence vibrations of $\mathrm{O}-\mathrm{H}$ and $\mathrm{C}-\mathrm{O}$ bonds. In our spectra it is mainly the vibrations of the transconformer which appear. It is also possible to state unequivocally that a small number of gauche-conformers is also present in the test sample. This is indicated by the presence of three bands in the region of the valence $\mathrm{O}-\mathrm{H}$ vibrations of the free hydroxyl group, as well as the presence of the bands at 1244,1060 , and $1057.5 \mathrm{~cm}^{-1}$, which, according to our calculations and Refs. 46 and 50, are the most intense absorption bands in the spectrum of the gauche-conformer of ethanol and are caused by valence vibrations of the $\mathrm{C}-\mathrm{C}-\mathrm{O}$ skeleton of the molecule.

\subsection{Interpretation of the FTIR spectra of ethyl alcohol in argon isolation}

We now examine the low-frequency vibrations of ethanol in more detail. The molecule contains two internal tops, with which these vibrations are associated. The harmonic approximation is less suitable for describing them. As the literature shows, the anharmonic approximation is capable, in principle, of providing an adequate estimate of the frequencies of the fundamental torsional vibrations. In the harmonic approximation the frequencies of the fundamental torsional vibrations of the hydroxyl and methyl groups for the transand gauche-conformers were 279.4; 235.1 and 272.2; $257.6 \mathrm{~cm}^{-1}$, respectively. Taking the anharmonicity into account yields $200.1 ; 215.1$ and $212.5 ; 233.6 \mathrm{~cm}^{-1}$. Our calculations show that the torsional vibrations of the hydroxyl and methyl groups are strongly shifted, especially for the trans-conformer. The most complete picture of the frequencies of the torsional vibrations of ethanol can be obtained using data from microwave spectra. ${ }^{32}$ The frequency of the fundamental torsional vibration of the hydroxyl group in the trans-conformer is $202.6 \mathrm{~cm}^{-1}$ and in the methyl, $244.4 \mathrm{~cm}^{-1}$. The attribution of the corresponding vibrations to the gauche-conformer is not so obvious, since the ground state is split into two sublevels separated by only $3.275 \mathrm{~cm}^{-1}$. The lower level corresponds to a linear combination of two gauche-isomers that is symmetric with respect to the C-C-O plane $\left(\mathrm{e}_{1}\right)$ and the upper level, to an antisymmetric combination $\left(\mathrm{o}_{1}\right)$. Perhaps the transition from the $\mathrm{e}_{1}$ state, which corresponds to a frequency of $243 \mathrm{~cm}^{-1}$ for the vibration of the hydroxyl group, should be regarded as the more "fundamental," but sometimes the transition from $\mathrm{o}_{1}$, for which the frequency is $195.8 \mathrm{~cm}^{-1}$, is combined with this. The $274 \mathrm{~cm}^{-1}$ band $^{46}$ recorded in an IR spectrum of ethanol in the gaseous phase has been attributed to the fundamental torsional vibration of the methyl group in the gauche-conformer. The excited states for the trans- and gauche-conformers are formally described by a pair of quantum numbers, $v_{\mathrm{QH}}=1, v_{\mathrm{CH}_{3}}=0$ and $v_{\mathrm{QH}}=0, v_{\mathrm{CH}_{3}}=1$, but the interrelation of the torsional states of the $\mathrm{OH}$ and $\mathrm{CH}_{3}$ groups is expressed through the delocalization of the wave functions of the excited states. An adequate description of the torsional motion of the two tops is possible only with a two-dimensional model. ${ }^{59}$ However, the calculations show that the motion of the hydroxyl group is essentially unperturbed by the motion of the methyl group. The motion of the latter is perturbed by the motion of the hydroxyl group, although the variations in the frequencies are small. This most likely is an indication that the interaction of these two vibrations is not as great as predicted by our calculations and by calculations in the MP2/cc-pVTZ approximation. ${ }^{64}$

According to the calculations in the harmonic approximation MP2/6-31G(d), the interaction among the vibrations is negligible (the contributions to the potential energy distribution (PED) for the trans- and gauche-conformers are $82 \% \tau_{\mathrm{OH}}+15 \% \tau_{\mathrm{CH}_{3}} ; 81 \% \tau_{\mathrm{CH}_{3}}+16 \% \tau_{\mathrm{OH}}$ and $89 \% \tau_{\mathrm{CH}_{3}}$; $91 \% \tau_{\mathrm{OH}}$. Calculations employing the spectroscopic mass of the hydrogen atom for the hydroxyl and methyl groups yield the following values for the fundamental frequencies of the vibrations: $207.3 ; 256.4 \mathrm{~cm}^{-1}$ (trans-conformer) and 234.8 ; $259.9 \mathrm{~cm}^{-1}$ (gauche-conformer). It can be said that neither the harmonic nor the anharmonic approximations give a satisfactory description of the large-amplitude intramolecular motions that are undoubtedly involved in torsional vibrations for low and medium potential barriers. However, the B3LYP/cc-pVQZ approximation with the anharmonicity effects taken into account has yielded values for the frequencies of the torsional vibrations of the hydroxyl and methyl groups in the correct order that agree with experiment, both for both the gauche- and the trans-conformers (Table 6). The deformation vibrations of the molecular skeleton (C-C-O) appear to have slightly higher frequencies. The anharmonic frequencies of these vibrations in the trans- and gaucheconformers are essentially the same $\left(414.0\right.$ and $\left.415.5 \mathrm{~cm}^{-1}\right)$ and agree very well with the experimental value of $416.5 \mathrm{~cm}^{-1}$.

We now proceed to the interpretation of the medium frequency spectral range. The four deformation vibrations of $\mathrm{C}-\mathrm{H}$ bonds in the methyl group are classified as scissoring, wagging, twisting and rocking motions, which we shall designate, respectively, by $\delta_{\mathrm{sc}}^{\mathrm{CH}_{2}}, \delta_{\mathrm{wag}}^{\mathrm{CH}_{2}}, \delta_{\mathrm{tw}}^{\mathrm{CH}_{2}}, \delta_{\text {rock }}^{\mathrm{CH}_{2}} \cdot{ }^{65}$ The frequencies of these vibrations usually fall off in the order listed here. The calculations show that the rocking vibrations of $\mathrm{C}-\mathrm{H}$ bonds in the methylene group are strongly shifted with the analogous vibrations of the $\mathrm{C}-\mathrm{H}$ bonds in the methyl group and form a low-intensity band at $812.0 \mathrm{~cm}^{-1}$. The calculated frequency of this vibration in the trans-conformer $\left(815.1 \mathrm{~cm}^{-1}\right)$ is closer to the experimental value than the frequency of the analogous vibration in the gauche-conformer $\left(804.2 \mathrm{~cm}^{-1}\right)$. The calculated intensity of the $825.1 \mathrm{~cm}^{-1}$ vibration is also very small. The calculations show that the $\delta_{\mathrm{sc}}^{\mathrm{CH}_{2}}$ vibration is also mixed with the deformation scissors 
TABLE 6. Comparison of calculated and observed vibrational frequencies of the ethanol molecule.

\begin{tabular}{|c|c|c|c|c|c|}
\hline \multicolumn{2}{|c|}{ Experiment } & & \multicolumn{2}{|c|}{ Calculated } & \multirow[b]{2}{*}{ Potential energy distribution } \\
\hline$\tilde{v}, \mathrm{~cm}^{-1}$ & $I, \mathrm{~km} / \mathrm{mole}$ & & $\tilde{v}, \mathrm{~cm}^{-1}$ & $I, \mathrm{~km} / \mathrm{mole}$ & \\
\hline 195.8 (Ref. 46) & & Gauche & 212.5 & 59.0 & $65.2 \% \tau_{1}+13.9 \% \tau_{2}$ \\
\hline 202.6 (Ref. 46) & & Trans & 200.1 & 78.0 & $62.2 \% \tau_{1}+22.3 \% \tau_{2}$ \\
\hline 243.1 (Ref. 46) & & Gauche & 212.5 & 59.0 & $65.2 \% \tau_{1}+13.9 \% \tau_{2}$ \\
\hline 244.4 (Ref. 46) & & Trans & 215.1 & 68.7 & $40.7 \% \tau_{1}+52.5 \% \tau_{2}$ \\
\hline 274.0 (Ref. 46) & & Gauche & 233.6 & 14.0 & $23.8 \% \tau_{1}+60.6 \% \tau_{2}$ \\
\hline 416.5 (Ref. 50) & & Trans & 414.0 & 10.0 & $\begin{array}{l}27.1 \% \gamma+7.9 \% \theta+8 \% \delta_{1}+8 \% \delta_{2}+8.4 \% \alpha_{1} \\
\quad+8.4 \% \alpha_{2}+4.4 \% \varphi+4.4 \% \beta_{1}+4.4 \% \beta_{2}\end{array}$ \\
\hline 651.0 & 0.01 & Dimer & & & \\
\hline 812.0 & 0.005 & Trans & 815.1 & 0.15 & $\begin{array}{c}17.9 \% \delta_{2}+17.9 \% \delta_{3}+16.9 \% \beta_{1}+16.9 \% \beta_{2}+12.1 \% \tau_{2} \\
+3.8 \% \chi_{1}+3.8 \% \chi_{2}+3.7 \% \alpha_{1}+3.7 \% \alpha_{2}\end{array}$ \\
\hline $\begin{array}{l}886.5 \\
888.0\end{array}$ & $\begin{array}{c}0.1 \\
0.02\end{array}$ & Trans & 879.9 & 12.0 & $\begin{array}{c}20.0 \% \delta_{1}+17.5 \% Q_{\mathrm{CO}}+16.3 \% Q_{\mathrm{CC}}+8.6 \% \delta_{2} \\
+8.6 \% \delta_{3}+4.9 \% \beta_{1}+4.9 \% \beta_{2}+4.6 \% \chi_{3}\end{array}$ \\
\hline $\begin{array}{l}1016.0 \\
1025.6\end{array}$ & $\begin{array}{c}0.03 \\
0.6\end{array}$ & Trans & 1000.9 & 65.6 & $\begin{array}{c}21.5 \% Q_{\mathrm{CC}}+17.5 \% \theta+11.6 \% Q_{\mathrm{CO}}+10.6 \% \delta_{1} \\
+7.3 \% \delta_{2}+7.3 \% \delta_{3}+4.4 \% \alpha_{1}+4.4 \% \alpha_{2}+4.4 \% \gamma\end{array}$ \\
\hline 1054.0 & 0.01 & Dimer & & & \\
\hline 1057.5 & 0.03 & & & & \\
\hline \multirow[t]{2}{*}{1060.0} & 0.01 & Gauche & 1035.0 & 6.0 & $\begin{array}{c}21.6 \% Q_{\mathrm{CC}}+12.7 \% \delta_{2}+12.6 \% Q_{\mathrm{CO}}+11.7 \% \delta_{1} \\
+10.0 \% \theta+9.5 \% \alpha_{2}+5.1 \% \gamma+4.7 \% \chi_{2}+3.3 \% \delta_{3} \\
\left(\delta_{\text {rock }}^{\mathrm{CH}_{2}}+\delta_{\text {rock }}^{\mathrm{CH}_{3}}\right)+\left(23.8 \% \tau_{1}+60.6 \% \tau_{2}\right)\end{array}$ \\
\hline & & Gauche & 1036.1 & 47.7 & \\
\hline $\begin{array}{l}1076.6 \\
1083.0\end{array}$ & $\begin{array}{l}0.02 \\
0.02\end{array}$ & Gauche & 1051.3 & 72.2 & $\begin{array}{l}18.3 \% Q_{\mathrm{CO}}+16.3 \% Q_{\mathrm{CO}}+11.9 \% \delta_{2}+10.3 \% \delta_{1} \\
\quad+10.2 \% \alpha_{2}+, 7.1 \% \alpha_{1}+6.8 \% Q_{\mathrm{CC}}+3.3 \% \chi_{2}\end{array}$ \\
\hline $\begin{array}{l}1088.0 \\
1092.0\end{array}$ & $\begin{array}{c}0.01 \\
0.2\end{array}$ & Trans & 1078.5 & 23.9 & $\begin{array}{l}19.9 \% Q_{\mathrm{CO}}+17.8 \% \delta_{1}+8.4 \% \delta_{2}+8.4 \% \delta_{3}+7.3 \% \theta \\
+6.5 \% \gamma+4.5 \% Q_{\mathrm{CC}}+3.6 \% \alpha_{1}+3.6 \% \alpha_{2}+2.6 \% \chi_{1}\end{array}$ \\
\hline 1115.0 & 0.003 & Gauche & 1114.7 & 4.5 & $\begin{array}{c}16.2 \% \delta_{3}+13.6 \% \delta_{1}+12.9 \% \alpha_{1}+9.4 \% Q_{\mathrm{CO}}+8.1 \% \alpha_{2} \\
+7.1 \% \gamma+6.1 \% \theta+6.1 \% \chi_{1}+6.0 \% \tau_{1}\end{array}$ \\
\hline 1161.0 & 0.01 & Trans & 1152.8 & 3.5 & $\begin{array}{c}18.5 \% \alpha_{1}+18.5 \% \alpha_{2}+14.2 \% \delta_{2}+14.2 \% \delta_{3}+6.5 \% \chi_{1} \\
+6.5 \% \chi_{2}+5.7 \% \beta_{1}+5.7 \% \beta_{2}+4.7 \% \tau_{2}+3.8 \% \tau_{1}\end{array}$ \\
\hline 1239.0 & 0.6 & Trans & 1229.5 & 70.9 & $\begin{array}{l}26.9 \% \theta+8.9 \% \beta_{1}+8.9 \% \beta_{2}+7.8 \% \delta_{1}+5.9 \% \delta_{2}+5.9 \% \delta_{3} \\
\quad+5.6 \% \alpha_{1}+5.6 \% \alpha_{2}+4.7 \% Q_{\mathrm{CO}}+4.1 \% \chi_{1}+4.1 \% \chi_{2}\end{array}$ \\
\hline 1244.0 & 0.01 & Dimer & & & \\
\hline $\begin{array}{l}1252.0 \\
1255.0\end{array}$ & $\begin{array}{l}0.02 \\
0.02\end{array}$ & Gauche & 1247.8 & 11.9 & $\begin{array}{c}16.6 \% \beta_{2}+13.3 \% \theta+12.3 \% \beta_{1}+10.8 \% \delta_{3}+8.5 \% \alpha_{2} \\
+6.2 \% \chi_{2}+5.8 \% \chi_{1}+4.8 \% \alpha_{1}+4.8 \% \tau_{2}+4.5 \% \tau_{1}\end{array}$ \\
\hline $\begin{array}{l}1369.0 \\
1372.0\end{array}$ & $\begin{array}{l}0.02 \\
0.02\end{array}$ & Trans & 1373.4 & 2.1 & $\begin{array}{c}15.4 \% \delta_{1}+15.0 \% \chi_{1}+15.0 \% \chi_{2}+12.9 \% \delta_{2}+12.9 \% \delta_{3} \\
+11.4 \% \chi_{3}+4.7 \% \beta_{1}+4.7 \% \beta_{2}+4.5 \% \alpha_{1}+4.5 \% \alpha_{2}\end{array}$ \\
\hline $\begin{array}{l}1412.0 \\
1415.0\end{array}$ & $\begin{array}{l}0.01 \\
0.01\end{array}$ & Trans & 1446.9 & 11.2 & $\begin{array}{c}12.3 \% \beta_{1}+12.3 \% \beta_{2}+12.1 \% \alpha_{1}+12.1 \% \alpha_{2}+12.0 \% \theta \\
+8.3 \% Q_{\mathrm{CC}}+6.9 \% \chi_{1}+6.9 \% \chi_{2}+4.1 \% \delta_{2}+4.1 \% \delta_{3}\end{array}$ \\
\hline 1444.7 & 0.03 & Trans & 1459.6 & 5.7 & $28.9 \% \chi_{1}+28.9 \% \chi_{2}+21.1 \% \tau_{2}+8.2 \% \delta_{2}+8.2 \% \delta_{3}$ \\
\hline \multirow[t]{3}{*}{$\begin{array}{l}1461.0 \\
1462.7\end{array}$} & 0.02 & Trans & 1475.1 & 2.8 & $\begin{aligned} 32.4 \% \varphi+ & 10.2 \% \chi_{3}+9.5 \% \alpha_{1}+9.5 \% \alpha_{2}+5.9 \% \alpha_{2}+5.9 \% \beta_{1} \\
& +5.9 \% \beta_{2}+4.1 \% \chi_{1}+4.1 \% \chi_{2}+3.5 \% \delta_{1}\end{aligned}$ \\
\hline & & Gauche & 1476.9 & 1.7 & $\begin{aligned} 27.6 \% \chi_{3} & +18.6 \% \varphi+9.5 \% \chi_{1}+8.6 \% \chi_{2}+8.6 \% \chi_{2}+8.6 \% \delta_{1} \\
& +7.7 \% \beta_{1}+6.9 \% \delta_{3}+3.3 \% \beta_{2}+3.2 \% \delta_{2}\end{aligned}$ \\
\hline & & Gauche & 1477.0 & 0.7 & $\begin{array}{c}30.9 \% \varphi+13.5 \% \alpha_{3}+9.7 \% \alpha_{2}+9.6 \% \alpha_{1}+9.5 \% \beta_{1}+5.9 \% \beta_{1} \\
+7.9 \% \beta_{2}+6.7 \% \alpha_{2}+5.8 \% \chi_{1}+5.2 \% \delta_{1}\end{array}$ \\
\hline $\begin{array}{l}1487.5 \\
1490.0\end{array}$ & $\begin{array}{c}0.015 \\
0.02\end{array}$ & Trans & 1484.0 & 1.5 & $\begin{array}{c}29.3 \% \chi_{3}+12.2 \% \chi_{1}+12.2 \% \chi_{2}+9.7 \% \varphi+8.8 \% \delta_{2} \\
+8.8 \% \delta_{3}+4.8 \% \beta_{1}+4.8 \% \beta_{2}\end{array}$ \\
\hline
\end{tabular}

vibrations of the $\mathrm{C}-\mathrm{H}_{8}$ and $\mathrm{C}-\mathrm{H}_{9}$ bonds in the methyl groups in opposite phase. As a result, in both conformers a pair of vibrations develop with a low frequency compared to the other deformation vibrations of the $\mathrm{C}-\mathrm{H}$ bonds. In the harmonic approximation, the vibration with a dominant contribution of $\delta_{\mathrm{sc}}^{\mathrm{CH}_{2}}$ to the PED (more than 65\%) lies higher for both conformers. However, when the anharmonicity is taken into account, the frequencies of these vibrations become essentially the same $\left(1477.0\right.$ and $\left.1476.9 \mathrm{~cm}^{-1}\right)$ in the gauche-conformer, while the $1484.1 \mathrm{~cm}^{-1}$ vibration with a dominant contribution from the methyl group (more than $55 \%)$ lies higher in the trans-conformer. The calculated frequency of the second vibration, in which the contribution of $\delta_{\mathrm{sc}}^{\mathrm{CH}_{2}}$ reaches $67 \%$, was $1475.1 \mathrm{~cm}^{-1}$. Since the anharmonic approximation has yielded a correct order of the sequence of the torsional vibrations of the hydroxyl and methyl groups in the trans- and gauche-conformers, we are inclined to attribute the $1487.5 ; 1490.0 \mathrm{~cm}^{-1}$ doublet to the $1484.1 \mathrm{~cm}^{-1}$ vibration and the $1461 ; 1462.7 \mathrm{~cm}^{-1}$ doublet to the $1475.1 ; 1476.9$ and $1477.0 \mathrm{~cm}^{-1}$ vibrations in the transand gauche-conformers even though these bands are assigned oppositely elsewhere. ${ }^{46,48,50}$

According to Table 5 the next vibration in terms of frequency for both conformers is the antisymmetric 
deformation vibration of the $\mathrm{C}-\mathrm{H}$ bonds in the methyl group, which is conveniently designated as $\delta_{\text {as }}^{\mathrm{CH}_{3}}(E)$ (the subscript indicates the symmetry with respect to the C-C-O plane and the symbol in parentheses, the type of symmetry with respect to the local $C_{3 V}$ group). Thus, the $1447.7 \mathrm{~cm}^{-1}$ band is attributed to this vibration in the trans-conformer $\left(1459.6 \mathrm{~cm}^{-1}\right)$, in full agreement with published data. ${ }^{46,48,50}$ The calculations show that the $\delta_{\text {wag }}^{\mathrm{CH}_{2}}$ vibration lies even lower. The frequencies of this vibration $\left(1446.9\right.$ and $1389.9 \mathrm{~cm}^{-1}$, respectively) differ substantially between the trans- and gauche-conformers. This is because of a contribution to the PED from deformation vibrations of the hydroxyl group, $\delta_{\mathrm{OH}}$. Because of a difference in its orientation relative to the $\mathrm{C}-\mathrm{C}-\mathrm{O}$ atoms, the reduced mass and force constant of these normal modes vary. The $1412.0 ; 1415 \mathrm{~cm}^{-1}$ band doublet is assigned to this vibration in the trans-conformer, in accord with Refs. 46 and 50 . The $1415 \mathrm{~cm}^{-1}$ band is mistakenly attributed to $\delta_{\text {rock }}^{\mathrm{CH}_{2}}$ in Ref. 48 .

It is worth noting that the $1389.1 \mathrm{~cm}^{-1}$ band is attributed to $\delta_{\text {wag }}^{\mathrm{CH}_{2}}$ in Ref. 46, in outstanding agreement with our calculations. The next band doublet $\left(1369.0\right.$ and $\left.1372.0 \mathrm{~cm}^{-1}\right)$ is undoubtedly caused by characteristic fully symmetric deformations of the $\mathrm{C}-\mathrm{H}$ bonds in the methyl group $\left(\delta_{s}^{\mathrm{CH}_{3}}\left(A_{1}\right)\right)$. The calculated frequencies of this vibration in the trans- and gauche-conformers are similar (1373.4 and $1376.3 \mathrm{~cm}^{-1}$ ) and are in outstanding agreement with experiment.

Analogous attributions have been proposed in Refs. 46, 48 , and 50 . A $1252.0 ; 1255.0 \mathrm{~cm}^{-1}$ doublet is observed in the lower frequency range. This doublet did not appear in a low-temperature IR spectrum of the trans-conformer of ethanol in an argon matrix in Ref. 50. At the same time, bands of trans-ethanol in a nitrogen matrix were observed in that paper at 1274.8 and $1256.3 \mathrm{~cm}^{-1}$. The first band is attributed to $\delta_{\mathrm{tw}}^{\mathrm{CH}_{2}}$ and the second to $\delta_{\mathrm{OH}}$ in the trans-conformer. Perchard attributes the bands at 1337.3 and $1260.6 \mathrm{~cm}^{-1}$ in the IR spectrum of ethanol in an $\mathrm{N} 2$ matrix to $\delta_{\mathrm{tw}}^{\mathrm{CH}_{2}}+\delta_{\mathrm{wag}}^{\mathrm{CH}_{2}}+\delta_{\mathrm{OH}}$ and $\delta_{\mathrm{tw}}^{\mathrm{CH}_{2}}+\delta_{\mathrm{OH}}$ vibrations, respectively, of the gaucheconformer of ethanol. Barnes ${ }^{48}$ recorded a band at $1250.8 \mathrm{~cm}^{-1}$ in the low-temperature IR spectrum of ethanol in an argon matrix and attributed it to $\delta_{\mathrm{tw}}^{\mathrm{CH}_{2}}$. Durig ${ }^{46}$ associates the $1272.0 \mathrm{~cm}^{-1}$ band with $\delta_{\mathrm{tw}}^{\mathrm{CH}_{2}}$ vibrations in the transconformer of ethanol in a low-temperature xenon solution. According to the calculations, the $\delta_{s}^{\mathrm{CH}_{3}}\left(A_{1}\right)$ vibrations of the trans-conformer are actually followed by $\delta_{\mathrm{tw}}^{\mathrm{CH}_{2}}\left(1267.9 \mathrm{~cm}^{-1}\right)$ with a contribution of up to $63 \%$ to the PED. Here there is no contribution whatever from deformation vibrations of the hydroxyl group. Next and lower, at a frequency of 1229.5 $\mathrm{cm}^{-1}$, there is a vibration with a predominant contribution of $27 \%$ from $\delta_{\mathrm{OH}}$. The remaining contribution here is from wagging vibrations of the $\mathrm{C}-\mathrm{H}$ bonds of the methylene and methyl groups. In the gauche-conformer the calculated vibrational frequencies in this spectral interval are 1328.9 and $1247.8 \mathrm{~cm}^{-1}$. The two vibrational modes are close in form and have somewhat different contributions from $\delta_{\mathrm{OH}}$ and $\delta_{\mathrm{tw}}^{\mathrm{CH}_{2}}$, while the first also has a significant contribution from $\delta_{\text {wag }}^{\mathrm{CH}_{2}}$.

Based on these data and noting the fact that the vibrations at $1267.9 \mathrm{~cm}^{-1}$ have a very low intensity according to the calculations (Table 5) while the vibrations at $1247.8 \mathrm{~cm}^{-1}$ have a relatively high intensity, we relate the $1252.0 ; 1255.0 \mathrm{~cm}^{-1}$ band doublet to the vibrations at $1247.8 \mathrm{~cm}^{-1}$ in the gauche- conformer of ethanol. In fact, the twisting vibrations of the $\mathrm{C}-\mathrm{H}$ bonds in the $\mathrm{CH}_{2}$ group of the trans-conformer do not cause any change in the dipole moment of the molecule, either in magnitude or direction, so the vibrations at $1267.9 \mathrm{~cm}^{-1}$ are essentially inactive in the IR spectrum. At the same time, the vibrations at $1247.8 \mathrm{~cm}^{-1}$ in the gauche-conformer owing to the contribution of $\delta_{\mathrm{OH}}$ turn out to be extremely active in the IR spectrum. We are inclined to attribute the low intensity band at $1244.0 \mathrm{~cm}^{-1}$, whose intensity is somewhat higher in the sample with concentrations of 1:1000, to the dimer. The next absorption band at $1239.0 \mathrm{~cm}^{-1}$ is the most intense in the IR spectrum of ethanol should undoubtedly be attributed to the $1229.5 \mathrm{~cm}^{-1}\left(\delta_{\mathrm{OH}}\right)$ vibration of the trans-conformer, whose intensity was calculated to be the highest in the IR spectrum. The low-intensity $1161.0 \mathrm{~cm}^{-1}$ band was noticed in Ref. 50 but not counted among the fundamental bands in Ref. 48. According to Ref. 50, this band is caused by rocking vibrations of the $\mathrm{C}-\mathrm{H}$ bonds in the methyl and methylene groups of the trans-conformer. A band at $1156.0 \mathrm{~cm}^{-1}$ in a xenon solution has been attributed to this vibration of the transconformer. ${ }^{46}$ According to the calculations, the deformation vibrations of the hydroxyl group in the trans-conformer are followed by vibrations at $1152.8 \mathrm{~cm}^{-1}$ with equal contributions from $\delta_{\text {rock }}^{\mathrm{CH}_{2}}$ and $\delta_{\text {rock }}^{\mathrm{CH}_{3}}$. The analogous vibrations in the gauche-conformer have a frequency of $1114.7 \mathrm{~cm}^{-1}$ and an additional $\delta_{\mathrm{OH}}$ contribution. We associate these vibrations with the 1156.0 and $1115 \mathrm{~cm}^{-1}$ bands. The remaining bands in the IR spectrum of ethanol are related primarily to valence vibrations of the molecular skeleton. The 1092.0; $1088.8 \mathrm{~cm}^{-1}$ band doublet is caused by vibrations at $1078.5 \mathrm{~cm}^{-1}$ in the trans-conformer. Here the main contribution to the PED is from valence vibrations of the C-O bond and, slightly less, from counterphase valence vibrations of the $\mathrm{C}-\mathrm{C}$ bond. There is also a $\delta_{\mathrm{OH}}$ contribution. A similar attribution was proposed before. ${ }^{46,48,50}$

Similar vibrations in the gauche-conformer at a frequency of $1051.3 \mathrm{~cm}^{-1}$ and a substantially larger $\delta_{\mathrm{OH}}$ contribution evidently cause the $1076.6 ; 1083.0 \mathrm{~cm}^{-1}$ band doublet in the IR spectrum of ethanol. According to the calculations, these vibrations are the most intense in the IR spectrum of the gauche-conformer and, since less intense bands of the gaucheconformer were also observed in this spectrum of the sample, its appearance in the spectrum is entirely to be expected. Another antiphase vibrational mode of the $\mathrm{C}-\mathrm{O}$ and $\mathrm{C}-\mathrm{C}$ bonds in the trans- and gauche-conformers, but now with predominance of the $\mathrm{C}-\mathrm{C}$ bond contribution to the PED, according to the calculations, has frequencies of 1000.9 and $1035.0 \mathrm{~cm}^{-1}$, respectively. In both cases there is an additional $\delta_{\mathrm{OH}}$ contribution; in the first case it is twice as large as in the second. Because of this, the calculated intensity of the vibrations at $1000.9 \mathrm{~cm}^{-1}$ in the trans-conformer seems very high, so it can be reliably attributed to the second most intense absorption band in the IR spectrum of ethanol at $1025.6 ; 1016.0 \mathrm{~cm}^{-1}$. Vibrations at $1035.0 \mathrm{~cm}^{-1}$ together with composite vibrations at $1036.1 \mathrm{~cm}^{-1}$, enhanced by a Fermi resonance, produce the low-intensity band at $1060.0 \mathrm{~cm}^{-1}$. The in-phase vibrations of the $\mathrm{C}-\mathrm{O}$ and $\mathrm{C}-\mathrm{C}$ bonds in the trans- and gauche-conformers have similar shapes and frequencies: 879.9 and $866.6 \mathrm{~cm}^{-1}$. According to the calculations, it should show up with a medium intensity in the IR spectrum, so it can be reliably associated with the medium-intensity $888.0 ; 886.5 \mathrm{~cm}^{-1}$ doublet 
band. These attributions are fully consistent with earlier work. ${ }^{46,48,50}$ We attribute the $1057.5 ; 1054.0 \mathrm{~cm}^{-1}$ doublet and the band at $651.0 \mathrm{~cm}^{-1}$ to vibrations of the ethanol dimer. The complete interpretation of the IR spectrum of the ethanol molecule in an argon matrix is given in Table 6.

\section{Conclusion}

Low-temperature FTIR spectra of ethanol isolated in an argon matrix have been obtained for a range of temperatures in the $20-45 \mathrm{~K}$ range with concentration ratios of $1: 1000$ and 1:2000. A comparative analysis of these spectra and comparisons with published low-temperature spectra of ethanol have made it possible to determine the bands responsible for absorption by impurities and associates. Calculations of the structure of the gauche- and trans-conformers of ethanol in the B3LYP/ cc-pVQZ approximation have made it possible to reproduce the difference in the energies of the conformers and a number of structural and geometric parameters of the molecules in close agreement with experimental data. For the first time, anharmonic calculations of the IR spectra of the trans- and gauche-conformers of $\mathrm{CH}_{3} \mathrm{CH}_{2} \mathrm{OH}$ in the B3LYP/cc-pVQZ approximation have yielded an improved interpretation of the IR spectra and made it possible to account for the effect of Fermi resonances on their formation. It has been shown that in an argon matrix ethanol is predominantly in the transconfiguration, although the most intense absorption lines of the gauche-conformer were still observed in the spectra of the samples. Good agreement between the calculated and experimental values of the frequencies was obtained, as well as almost complete agreement regarding their sequence order, without scaling of the harmonic force fields. It was found that torsional vibrations of the methyl and hydroxyl groups are subject to anharmonicity to the greatest extent. We note that the calculations using harmonic force fields and the spectroscopic masses for the hydrogen atoms yielded fully competitive results (Table 1) while consuming considerably less time.

This work was supported by the State Foundation for Basic Research of Ukraine (Grant No. F41/138-2011) and the Belarusian Republic Foundation for Basic Research (Grant No. F11K-032).

a)Email: dori11@ukr.net

${ }^{1}$ V. Pogorelov, I. Doroshenko, P. Uvdal, V. Balevicius, and V. Sablinskas, Mol. Phys. 108, 2165 (2010).

${ }^{2}$ I. Doroshenko, V. Pogorelov, V. Sablinskas, and V. Balevicius, J. Mol. Liq. 157, 142 (2010).

${ }^{3}$ G. A. Pitsevich, I. Yu. Doroshenko, V. Ye. Pogorelov, and D. S. Umrejko, J. Spectrosc. Dyn. 1, 9 (2011).

${ }^{4}$ K. T. Hecht and D. M. Dennison, J. Chem. Phys. 26, 31 (1957).

${ }^{5}$ C. C. Lin and J. D. Swalen, Rev. Mod. Phys. 31, 841 (1959).

${ }^{6}$ G. A. Pitsevich and M. Shundalau, J. Spectrosc. Dyn. 2, 15 (2012).

${ }^{7}$ B. Zuckerman, B. E. Turner, D. R. Johnson, and F. O. Clark, Astrophys. J. Lett. 196, L99 (1975).

${ }^{8}$ T. J. Millar, H. Olafson, A. Hjalmarson, and P. D. Broun, Astron. Astrophys. 205, L5 (1988).

${ }^{9}$ T. J. Millar, G. H. Macdonald, and R. J. Habing, Mon. Not. R. Astron. Soc. 273, 25 (1995).

${ }^{10}$ P. Caselli, T. I. Hasegawa, and E. Herbst, Astrophys. J. 408, 548 (1993).

${ }^{11}$ B. Bak, E. S. Knudsen, and E. Madsen, Phys. Rev. 75, 1623 (1949).

${ }^{12}$ L. M. Imanov and Ch. O. Kadzhar, Opt. Spectrosc. 14, 157 (1963).

${ }^{13}$ J. Michielsen-Effinger, Bull. Cl. Sci., Acad. Roy. Belg. 50, 645 (1964).

${ }^{14}$ M. Takano, Y. Sasada, and T. Satoh, J. Mol. Spectrosc. 26, 157 (1968).

${ }^{15}$ Y. Sasada, M. Takano, and T. Satoh, J. Mol. Spectrosc. 38, 33 (1971).
${ }^{16}$ Ch. O. Kadzhar, I. D. Isaev, and L. M. Imanov, J. Struct. Chem. 9, 375 (1968).

${ }^{17}$ L. M. Imanov, A. A. Abdurakhmanov, and R. A. Ragimova, Opt. Spectrosc. 17, 162 (1964).

${ }^{18}$ L. M. Imanov, Ch. O. Kadzhar, and I. D. Isaev, Opt. Spectrosc. 18, 508 (1965).

${ }^{19}$ L. M. Imanov, Ch. O. Kadzhar, and I. D. Isaev, Opt. Spectrosc. 22, 454 (1967).

${ }^{20}$ L. M. Imanov, Ch. O. Kadzhar, and I. D. Isaev, Opt. Spectrosc. (Suppl. III), 169 (1968).

${ }^{21}$ J. Michielsen-Effinger, J. Mol. Spectrosc. 29, 489 (1968).

${ }^{22}$ J. Michielsen-Effinger, Bull. Cl. Sci., Acad. Roy. Belg. 53, 226 (1967).

${ }^{23}$ R. K. Kakar and P. J. Seibt, J. Chem. Phys. 57, 4060 (1972).

${ }^{24}$ C. R. Quade and C. C. Lin, J. Chem. Phys. 38, 540 (1963).

${ }^{25}$ R. K. Kakar and C. R. Quade, J. Chem. Phys. 72, 4300 (1980).

${ }^{26}$ T. J. Millar, G. H. Macdonald, and R. J. Habing, Mon. Not. R. Astron. Soc. 273, 25 (1995).

${ }^{27}$ P. Caselli, T. I. Hasegawa, and E. Herbst, Astrophys. J. 408, 548 (1993).

${ }^{28}$ J. C. Pearson, K. V. L. N. Sastry, E. Herbst, and F. C. D. Lucia, J. Mol. Spectrosc. 175, 246 (1996).

${ }^{29}$ C. F. Su and C. R. Quade, J. Mol. Spectrosc. 188, 1 (1998).

${ }^{30}$ R. D. Suenram, F. J. Lovas, C. R. Quade, and C. F. Su, J. Mol. Spectrosc. 188, 9 (1998).

${ }^{31}$ C. R. Quade, J. Mol. Spectrosc. 203, 200 (2000).

${ }^{32}$ J. C. Pearson, C. S. Brauer, and B. J. Drouin, J. Mol. Spectrosc. 251, 394 (2008).

${ }^{33}$ F. Weinhold, J. Chem. Phys. 109, 367 (1998).

${ }^{34}$ F. Weinhold, J. Chem. Phys. 109, 373 (1998).

${ }^{35}$ R. Ludwig, F. Weinhold, and T. C. Farrar, Mol. Phys. 97, 465 (1999).

${ }^{36} \mathrm{P}$. Borowski, J. Jaroniec, T. Janowski, and K. Wolinski, Mol. Phys. 101, 1413 (2003).

${ }^{37}$ V. Pogorelov, A. Yevglevsky, I. Doroshenko, L. Berezovchuk, and Yu. Zhovtobryuch, Superlattices. Microstruct. 44, 571 (2008).

${ }^{38}$ K. J. Gaffney, P. H. Davis, I. R. Piletic, N. E. Levinger, and M. D. Faeye, J. Chem. Phys. 106, 12012 (2002).

${ }^{39}$ K. J. Gaffney, I. R. Piletic, and M. D. Fayer, J. Phys. Chem. 106, 9428 (2002).

${ }^{40}$ M. A. F. H. van der Broek, H. K. Nienhuys, and H. J. Bakker, J. Chem. Phys. 114, 3182 (2001).

${ }^{41}$ M. Abu-samba, K. J. Borve, L. J. Sethre, and T. D. Thomas, Phys. Rev. Lett. 95, 103002 (2005).

${ }^{42}$ W. Ritchter, D. Schiel, and W. Woger, Mol. Phys. 60, 691 (1987).

${ }^{43}$ Y. Mikawa, J. W. Brasch, and R. J. Jakobsen, Spectrochim. Acta 27A, 529 (1971).

${ }^{44}$ J. R. Durig and C. W. Hawley, J. Phys. Chem. 75, 3993 (1971).

${ }^{45}$ J. R. Durig, W. E. Bucy, and C. J. Wurrey, J. Phys. Chem. 79, 988 (1975).

${ }^{46}$ J. R. Durig, H. Deeb, I. D. Darkhalil, J. J. Klassen, and T. K. Gounev, J. Mol. Struct. 985, 202 (2011).

${ }^{47}$ J. P. Perchard and M. L. Josien, J. Chim. Phys. 65, 1834 (1968),

${ }^{48}$ A. J. Barnes and H. E. Hallam, Trans. Faraday Soc. 66, 1932 (1970).

${ }^{49}$ A. J. Barnes, J. Mol. Struct. 113, 161 (1984).

${ }^{50}$ S. Coussan, Y. Bouteiller, J. P. Perchard, and W. Q. Zheng, J. Phys. Chem. A 102, 5789 (1998).

${ }^{51}$ M. J. Frisch, G. W. Trucks, H. B. Schlegel, G. E. Scuseria, M. A. Robb, J. R. Cheeseman, J. A. Montgomery, Jr., T. Vreven, K. N. Kudin, J. C. Burant, J. M. Millam, S. S. Iyengar, J. Tomasi, V. Barone, B. Mennucci, M. Cossi, G. Scalmani, N. Rega, G. A. Petersson, H. Nakatsuji, M. Hada, M. Ehara, K. Toyota, R. Fukuda, J. Hasegawa, M. Ishida, T. Nakajima, Y. Honda, O. Kitao, H. Nakai, M. Klene, X. Li, J. E. Knox, H. P. Hratchian, J. B. Cross, V. Bakken, C. Adamo, J. Jaramillo, R. Gomperts, R. E. Stratmann, O. Yazyev, A. J. Austin, R. Cammi, C. Pomelli, J. W. Ochterski, P. Y. Ayala, K. Morokuma, G. A. Voth, P. Salvador, J. J. Dannenberg, V. G. Zakrzewski, S. Dapprich, A. D. Daniels, M. C. Strain, O. Farkas, D. K. Malick, A. D. Rabuck, K. Raghavachari, J. B. Foresman, J. V. Ortiz, Q. Cui, A. G. Baboul, S. Clifford, J. Cioslowski, B. B. Stefanov, G. Liu, A. Liashenko, P. Piskorz, I. Komaromi, R. L. Martin, D. J. Fox, T. Keith, M. A. Al-Laham, C. Y. Peng, A. Nanayakkara, M. Challacombe, P. M. W. Gill, B. Johnson, W. Chen, M. W. Wong, C. Gonzalez, and J. A. Pople, GAUSSIAN 03, Revision B. 05, Gaussian, Inc., Wallingford, CT, 2004.

${ }^{52}$ A. D. Becke, J. Chem. Phys. 98, 5648 (1993).

${ }^{53}$ C. Lee, W. Yang, and R. G. Parr, Phys. Rev. B 37, 785 (1988).

${ }^{54}$ P. J. Stephens, F. J. Devlin, C. F. Chabalowski, and M. J. Frisch, J. Phys. Chem. 98, 11623 (1994).

${ }^{55}$ T. H. Dunning, Jr., J. Chem. Phys. 90, 1007 (1989).

${ }^{56}$ G. A. Pitsevich, M. Shundalau, M. A. Ksenofontov, and D. S. Umreiko, Global J. Anal. Chem. 2, 114 (2011). 
${ }^{57}$ S. Califano, Vibrational States (Wiley, New York, 1976).

${ }^{58}$ G. Herzberg, Infrared and Raman Spectra (Van Nostrand, New York, 1945).

${ }^{59}$ I. M. Mills, in: Molecular Spectroscopy: Modern Research, edited by K. N. Rao and C. W. Mathews (Academic Press, New York, 1972), Vol. 1, p. 115 .

${ }^{60}$ J. C. Pearson, K. V. L. N. Sastry, M. Winnewisser, E. Herbst, and F. C. D. Lucia, J. Phys. Chem. 24, 1 (1995).

${ }^{61}$ J. J. Turner, Matrix Isolation Spectroscopy, edited by A. J. Barnes, W. J. Orville-Thomas, A. Muller, and R. Gaufres (D. Reidel, Dordrecht, 1981), p. 177.
${ }^{62}$ G. A. Pitsevich, I. Y. Doroshenko, V. E. Pogorelov, V. Shablinskas, V. Balevichus, and E. N. Kozlovskaya, J. Am. Chem. 2, 218 (2012).

${ }^{63}$ A. J. Barnes, Matrix Isolation Spectroscopy, edited by A. J. Barnes, W. J. Orville-Thomas, A. Muller and R. Gaufres (D. Reidel, Dordrecht, 1981), p. 18.

${ }^{64}$ M. L. Senent, Y. G. Smeyers, R. Dominguez-Gomez, and M. Villa, J. Chem. Phys. 112, 5809 (2000).

${ }^{65}$ N. P. G. Roeges, A Guide to the Complete Interpretation of the Infrared Spectra of Organic Structures (Wiley, New York, 1994).

Translated by D. H. McNeill 